\title{
Novel Technique for Coal Pyrolysis and Hydrogenation Product Analysis
}

Final Technical Report

March 15, 1993

Lisa D. Pfefferle, Associate Professor

German Bermudez, Research Associate James Boyle, Ph.D Studeni

GRANT NO.: DE-FG22-89PC89767

Yale University

Department of Chemical Engineering

P.O. Box 2159 Yale Station

New Haven, CT 06520.

(203) $432-2460$ 
FINAL TECHNICAL REPOR'T 3/93

\section{TABLE OF CONTENTS}

1.0 Objective

2.0 Summary of Work Done and Conclusions 2

3.0 Significance to Fossil Energy Program 3

$\begin{array}{lll}4.0 & \text { Results } & 3\end{array}$

4.1 Description of Experimental Details 3

4.1.1 The Microjet Reactor . 4

4.1.2 Time of Flight Mass Spectrometer 5

4.1.3 Optical Techniques for VUV Generation 6

4.2 Determination of Neutral Molecule Detection Efficiency 7

$\begin{array}{llll}\text { 4.2.1 General Issues } & 7\end{array}$

$\begin{array}{ll}\text { 4.2.2 Estimating VUV Photoionization Efficiency from Absorption } & \\ \text { and Photoelectron Spectroscopy Measurements } & 8\end{array}$

4.3 Discrimination of Isomeric Compounds $\quad 11$

4.3.1 Simultaneous Photoionization and Pulsed Electron Ionization 12

The UV-LDEI System $\quad 12$

The VUV-LDEI System 13

Summary Results from UV or VUV-LDEI

4.3.2 Improvements for Attaining Compound Selectivity
Suggested for Future Work

4.4 Ilydrocarbon Pyrolysis and Oxidation Combustion 16

4.4.1 IIigher IIydrocarbon Production during Allene Pyrolysis 16

Summary $\quad 16$

Background $\quad 16$

$\begin{array}{ll}\text { Experimental Procedure } & 18\end{array}$

Experimental Results and Discussion 18

Low Temperature Benzene Formation Mechanism . 21

lligh Temperature Benzene Formation 23

Iligher Ilydrocarbon Formation at Long Residence Times 24

$\begin{array}{ll}\text { Conclusion of the Allene Pyrolysis Study } & 27\end{array}$

4.4.2 Benzene Oxidation and the Oxidative Pyrolysis of Benzene 27

$\begin{array}{ll}\text { Procedure } & 28\end{array}$

Data Analysis $\quad 28$

Results and Discussion 31

Conclusion of Benzene Oxidation Study 34

5.0 Overall Technical Conclusion and Recommendations 35

6.0 Tables and Figures $\quad 36$

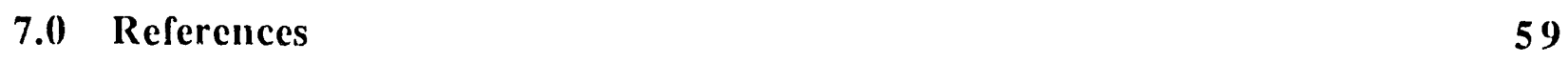

8.0 Ilighlight Accomplishments $\quad 61$

\begin{tabular}{l}
9.0 Articles and Presentations \\
\hline 1
\end{tabular} 


\section{Final Technical Report 3/15/93}

TITLE: Novel Technique for Coal Pyrolysis and Hydrogenation Product Analysis

PI: Lisa D. Pfefferle, Associate Professor

RESEARCH ASSOCIATE: German Bermudez

STUDENT(S) AND THE DEGREE FOR WHICH THEY ARE REGISTERED: James Boyle, Ph.D.

INSTITUTION/ORGANIZATION: Yale University, Department of Chemical Engineering, P.O. Box 2159 Yale Station, New Haven, CT 06520.

Phone number of PI: (203) 432-2460

GRANT NO.: DE-FG22-89PC89767

\subsection{OB.JECTIVE}

The overall objective of this study was to establish vacuum ultraviolet photoionization-MS and VUV pulsed EI-MS as useful tools for a simpler and more accurate direct mass spectrometric measurement of a broad range of hydrocarbon compounds in complex mixtures for ultimate application to the study of the kinetics of coal hydrogenation pyrolysis and oxidation processes. Issues related to the application of VUV-MS for monitoring and control of coal processing reactors were also addressed. The VUV-MS technique allows ionization of a broad range of species with minimal fragmentation. Many compounds of interest can be detected with the initial wavelength used $(118 \mathrm{~nm})$, but additional compound selectivity is achievable by tuning the wavelength of the photo-ionization source in the VUV. Other techniques for discrimination of molecular isomers such as series mass filters and meta-stable ion decay experiments have also been investigated as means to achieve compound selectivity. 


\subsection{SUMMARY OF VORK DONE AND CONCLUSIONS}

A microjet reactor coupled to a VUV photoionization time-of-flight mass spectrometer has been used to obtain species measurements during high temperature pyrolysis and oxidation of a wide range of hydrocarbon compounds ranging from allene and acetylene to cyclohexane, benzene and toluene. Initial work focused on calibration of the technique, optimization of ion collection and detection and characterization of limitations. Using the optimized technique with $118 \mathrm{~nm}$ photoionization, intermediate species profiles were obtained for analysis of the hydrocarbon pyrolysis and oxidation mechanisms. The "soft" ionization, yielding predominantly molecular ions, allowed the study of reaction pathways in these high temperature systems where both sampling and detection challenges are severe. Work has focused on the pyrolysis and oxidative pyrolysis of aliphatic and aromatic hydrocarbon mixtures representative of coal pyrolysis and hydropyrolysis products.

The detailed mass spectra obtained during pyrolysis and oxidation of hydrocarbon mixtures is especially important because of the complex nature of the product mixture even at short residence times and low primary reactant conversions. In addition, use of only single compound data to predict the pyrolysis characteristics of mixtures is not realistic by itself because reaction channels inyolving intermediates from pyrolysis of the more reactive hydrocarbon with the less reactive hydrocarbons fuel are not obvious. The combustion community has advanced detailed modeling of pyrolysis and oxidation to the $\mathrm{C} 4$ hydrocarbon level but in general above that size uncertainties in rate constant and thermodynamic data do not allow us to a priori predict products from mixed hydrocarbon pyrolyses using a detailed chemistry model. For pyrolysis of mixtures of coal-derived liquid fractions with a large range of compound structures and molecular weights in the hundreds of amu the modeling challenge is severe. Lumped nodels are possible from stable product data, but care must be taken in using these lumped models outside of the operating conditions where the data was obtained. In our work, wn are developing the VUV-MS technique as a diagnostic to allow building of detailed models and determining regions of applicability for lumped models. The work which we have completed under this project on the characterization of large PAH (100 - $1000 \mathrm{amu})$ during hydrocarbon pyrolysis is providing the basis for use of this diagnostic technique in less well characterized systems. 


\subsection{SIGNIFICANCE TO FOSSIL, ENERGY PRQGRAM}

This research is innovative, offering a relatively new technique with significantly improved performarice for the mass detection of $>C_{1}$ hydrocarbons in mixtures. This technique would facilitate the scientific investigation of coal upgrading processes such as pyrolysis and hydrogenation by allowing accurate direct analysis of both stable and intermediate reaction products. The on-line, non-destructive nature of the VUV-MS technique facilitates data collection and analysis, allowing more experiments to be run with greater analysis accuracy, greatly improving the database and allowing more confident prediction of reaction pathways.

\subsection{RESULTS}

The report below is organized in two main sections: 1) the description of experimental details which summarizes the technique development, calibration and selectivity considerations and 2) detailed analysis from examples of application to hydrocarbon reaction systenıs of interest to coal chemistry. Limitations of the technique in its current form are discussed along with evaluation of improvements for future work. Our most recent work towards application of VUV-MS for coal pyrolysis analysis is focused on developing theoretically-based correlation schemes for quantitative detection and means of obtaining product selectivity. The two hydrocarbon pyrolysis oxidation experiments described below demonstrate the power of the technique for studying hydrocarbon pyrolysis/growth mechanisms in complex high temperature systems.

\subsection{Description of Experimental Details}

Experiments were carried out in choked-flow continuous nozzle sources (microjet reactor) discharging directly into a vacuum chamber where the product species were ionized, collected and detected. Allene, benzene, toluene, acetylene and mixtures of the fuels were used both in the presence and absence of oxygen. The gas stream emanating from the sampled reactor or calibration source was ionized by the VUV radiation and resulting ions detected by the time-offlight (TOF) mass spectrometer. A TOF mass spectrometer was chosen because the entire mass spectra can be obtained with each laser ionization pulse, greatly improving the sensitivity of the system for multicomponent analysis. As a result, the TOF mass spectrum consists of a simple fingerprint, giving the masses of the components in the gas mixture 


\subsubsection{The Microjet Reactor}

The microjet reactor is a miniature fast flow reactor. It consists of one or more ceramic tubes through which reactants are passed and heated prior to adiabatic expansion through a pinhole opening. The outer most tube is used both for the confinement of the reaction and as the nozzle for ejection of intermediate species into the vacuum chamber for analysis. Nozzles vary between 50 and 200 microns in diameter. In operation, the microjet reactor is resistively heated to a desired calibrated wall temperature.

The microjet is both a combustion/pyrolysis reactor and molecular beam source. As a molecular beam source, it provides automatic and controlled sampling of the reactions taking place in the combustor via a pinhole orifice. The physical dimension of the orifice affects both the fluid dynamics of the reactor and the gas dynamics of the adiabatic expansion as reaction products are exhausted. Consequently, an orifice diameter is chosen to satisfy the following constraints:

1. A predetermined mass flux through the reactor at a given microjet pressure, yielding some suitably short mean residence time and high enough Re numbers for good heat and mass transport;

2. A predetermined, tolerable background vacuum pressure downstream of the microjet, allowing mean free paths on the order of the neutral or ion transit distance to the mass spectrometer;

3. A predetermined expansion of suitably short physical and temporal dimensions, to rapidly freeze kinetic processes carried out in the microjet.

Pumping constraints (on the order of 1000 liters per second) normally limits the choice of orifice diameter to something on the order of $0.1 \mathrm{~mm}$ or less. At the other extreme, nozzle diameters of $0.05 \mathrm{~mm}$ and less are difficult to obtain and more importantly, the reduced mass fluxes at these smaller orifice sizes result in extremely small Reynolds numbers impairing heat and mass transport and making residence time distribution difficult to characterize.

What makes the microjet especially attractive for studying combustion/pyrolysis reactions is the extremely short spatial and temporal dimensions necessary for adequately quenching reacting species as they are ejected from the reactor. A microjet reactor constructed with a $0.1 \mathrm{~mm}$ diameter nozzle will yield quenched species within 1-2 microseconds. These species will have undergone both rapid decreases in density ( $c a .0 .001$ times its source value) and temperature (ca 0.01 times its 
source value). This allows labile species such as hydrocarbon radicals to be at least semiquantitatively measured because their concentration is not perturbed from its upstream value. VUV ionization allows "non-perturbing" detection, so the combination of these two methodologies is particularly powerful in studying kinetic mechanisms where hydrocarbon radical processes play an important role such as high temperature coal pyrolysis.

Equally important, the expansion characteristics of the microjet ensure that the 'core' gas has a sufficiently high Reynolds number. Thus, the expanding gas is virtually free of wall interaction effects as it transits into the vacuum chamber. By comparison, sampling probes inserted into low pressure stabilized flames, by virtue of the size scale of the sampling orifice and the typical flow velocities encountered, are much less dependable for eliminating gas-wall interactions resulting in sample distortion.

We have carried out initial experiments on benzene oxidation in a microjet reactor to compare results directly with those obtained by Glassman and coworkers (e.g. Brezinsky et. al.) in the Princeton flow reactor system. This was done to find out how well microjet reactor data corresponded with that obtained under similar conditions in the flow reactor environment. In this study, major species profile and rate data agree quite well and from the nomalized spectra of relative concentrations and in addition to stable species, hydrocarbon radicals were also measured. This reaction system is described below in Section 4.4.2.

\subsubsection{Time of Flight Mass Spectrometer}

The time-of-flight spectrometer was constructed in our laboratories and is configured for use with UV and VUV laser ionization. It includes a TOF mass analyzer, a pumping system for analyzer, ionization and vaporization stages (each stage is valved so as to minimize down-time with sample changes), a reflectron, and power supplies for the ion reflector, Galileo HOT technology micro channel plates (MCP) detectors and ion sources. The Galileo HOT MCP plates provide an important improvement for ion detection as they were observed to provide a linear response from single ion counting through $\mathrm{mV}$ signals. This was not true for other MCP detector configurations tested. Combustion/pyrolysis emissions can include a considerable quantity of neutral polymeric hydrocarbon compounds which, if allowed to collect on the micro-channel plate ion detectors, would render it inoperative. This condition necessitates placing the neutra' suurce at a $90^{\circ}$ axis from the time of flight axis or using a source aimed into an ion mirror (reflectron) allowing ions to be reflected into the detector without neutral deposition. In the reported work, we have used both configurations. In the case of $90^{\circ}$ ion extraction, an Einzel lens is used to correct for the normal 
component of kinetic energy. Figures $1 \mathrm{a}$ and $1 \mathrm{~b}$ show schematics of the VUV TOF-MS showing the two configurations used for ion collection and detection.

The TOF mass spectrometer is ideal for our work with optimization of VUV ionization sources for the detection of hydrocarbon pyrolysis/combustion products. The system is designed for use with complementary spectroscopies, so many optical access ports are provided. The valving arrangement of the mass spectrometer was designed to allow us to make frequent sample changes and ionizer adjustments with minimal turnaround time due to the small pump down volume. This is critical for system optimization because it minimizes the delay in making system changes.

\subsubsection{Optical Techniques for VUV Generation}

VUV photons can be produced using laser light generated by the non-linear optical mixing technique of third harmonic generation (THG). Laser radiation, when focused into a gas with a resonance just to the red of the wavelength corresponding to a three photon absorption, will generate a single-photon with a wavelength equal to one-third the wavelength of the incoming photons, given satisfaction of phase matching conditions. One such gas with an accessible resonance is Xenon, which has a three photon resonance just to the red of $118.2 \mathrm{~nm}$ and at 107 $\mathrm{nm}$. Third harmonic generation in Xenon is the technique employed in this work mainly to produce the $118 \mathrm{~nm}$ photons.

Because of the niany attractive possible applications, research on generation of tunable vacuum ultraviolet radiation has been actively pursued in the past two decades. VUV radiation is absorbed by all solid optical materials, therefore, a wavelength limit for "windowless generation" of about $106 \mathrm{~nm}$ is imposed by the transmission cutoff of LIF and MgF lenses. VUV can be generated through resonantly enhanced $\chi^{3}$ processes in gases and metal vapors. A serious constraint is that difference frequency mixing schemes based on two-photon resonances inherently are restricted to energies to the red-side of the resonance energy. Sum frequency generation allows extension of this wavelength range but is rigidly limited to spectral regions of negative dispersion. This is a result of phase-matching requirements for focussed Gaussian beams. Difference frequency generation, on the other hand, is possible in regions of both positive and negative dispersion. A new technique developed by Strauss and Funk that allows generation of VUV photons to the practical lithium fluoricle cutoff employs four-wave difference frequency mixing. This technique allows semi-continuous tuning from 108-193 nm with reasonable efficiency. In future work we will use this technique to allow more flexible tuning of the VUV ionization wavelength in our hydrocarbon analyses. 
An important aspect of use of laser generated VUV photons for ionization is differential focusing of the UV and VUV photons. High fluxes of residual UV photons must not be allowed to interact with the sample volume, or multimhoton events and fragmentation will obscure the measurement. The two wavelengths can be separated by differential focusing taking advantage of differences in the refractive indices in the lens material as a function of wavelength. For the two lens materials with the greatest transmission efficiency, $\mathrm{LIF}$ and $\mathrm{MgF}_{2}$, only $\mathrm{MgF}_{2}$ offers a great enough refractive index difference to significantly spread the UV and VUV focal points and hence minimize UV interference with VUV studies. Using a $\mathrm{MgF}_{2}$ lens, the sample outlet is mounted $150 \mathrm{~mm}$ from the Xe cell, far from the focal point of the UV beam (at $355 \mathrm{~nm}$ ). This reduced the signal to below detection limits demonstrating little interference from $355 \mathrm{~nm}$ multiphoton ionization (MPI).

\subsection{Determination of Neutral Molecule Detection Efficiency}

\subsubsection{General Issues}

In order to perform quantitative measurements of neutral hydrocarbon pyrolysis products, each of the processes which a sampled molecule must undergo in order to be detected by the instrument must be characterized. The maximum measured detection efficiency is the product of each of the individual efficiencies associated with 1) the delivery of the sampled species to the ionization volume, 2) the ionization yield, 3) the efficiency of ion transmission through the spectrometer to an ion detector and the detector response to an incident charged molecule. It is important to both minimize and characterize species dependent components of the measurement efficiency.

Several different experimental/theoretical strategies have been developed in this work to characterize and optinuze detection efficiency. Issues related to relative photoionization efficiency are discussed in detail below. The other important components for achieving accurate species measurement are the relative efficiency of collecting ions from the ionization region and the relative detection efficiency for different species of the MCP detectors. In order to correct for kinetic energy in the direction nomal to the time of flight tube, an Einzel lens has been designed and optimized based on detection of mixtures representative of those expected in our experiments. This assures that all collected ions reach the detector at the end of the flight tube with approximately the same efficiency.

Relative detection efficiencies of the various molecular hydrocarbons must likewise be considered to obtain quantitative data. In the recent work of Geno and MacFarlane, an integrated probability of detection was calculated based upon the relative signals recovered from a variety of small 
pepticles $(\mathrm{m} / \mathrm{z} 86$ to $\mathrm{m} / \mathrm{z} 1059)$. We have tested this correlation in our own system, based upon the similarity of hydrocarbons and peptides. Accordingly, one may then compute a general secondary electron emission coefficient $(\gamma)$ by

$$
\gamma=2.58 \times 10^{-7} m\left[\exp \left(2.31 \times 10^{-4} v\right)\right]
$$

where $m$ is the molecular weight of the ion (amu) and $v$ is the velocity $(\mathrm{m} / \mathrm{s})$. From this value one may then compute the average probability for detecting an ion from the following:

$$
P=1-e^{-\gamma}
$$

Applying these relationships to our own experimental operating conditions ( $2-\mathrm{kV}$ postacceleration), the average probability of detecting hydrocarbon ions impacting on the micro-channel plate is essentially unity through $150 \mathrm{amu}$. For ions larger than approximately biphenyl, however, corrections must be applied in order to recover relative incident flux at the detector face.

\subsubsection{Estimating VUV Photoionization Efficiency from Absorption and Photoelectron Spectroscopy Measurements}

Strategies for estimating VUV photoionization efficiencies of hydrocarbons using both absorption techniques and photoelectron spectroscopy (PES) measurements have been developed by a variety of investigators to augment experimentally-based calibrations. In PES, an intense beam of photons (e.g. from the Helium resonance line at $58.4 \mathrm{~nm}(\mathrm{hv}=21.22 \mathrm{eV})$ ) is used to ionize electrons from the valence shell of the probed molecule. The irradiated molecule undergoing photoionization emits electrons which are counted as a function of their kinetic energy. This energy spectrum is a measure of the orbital character of the molecule. For cliatomic and larger species, excitation of either vibrational or rotational degrees of freedom upon ionization can lead to additional detected electrons. Multiple lines, each corresponding to a single vibrational transition, appear as bands of electron activity from a single molecular orbital. Larger polyatomic molecules have both more molecular orbitals from which ionization can take place, and numerous modes of vibration which may be excited complicating the photoelectron spectra. Due to the vibrational structure of the molecule, individual lines are not easily resolved. Line broadening also results from the short lifetimes of the individual molecular ionic states which are formed in the PES experiment. Polyatomic ions may either dissociate, radiate away their energy, or internally convert to another electronic state. Since the uncertainty principle dictates that the energy width scales as the inverse of the ionic state lifetime by $\Delta \mathrm{E} \approx \mathrm{h} / \Delta \mathrm{t}$, there is an additional line broadening of the spectral lines. Approximation methods are therefore required whe: interpreting these spectra to allow reasonable 
estimates of the ratio of the ionization cross section to absorption cross section for any given molecule. The VUV photoionization quantun yield will be a function of both the photon energy as well as the vibrational state (temperature) of the molecule.

To a first approximation, Eland, 1984 found that the areas of the individual bands in a photoelectron spectrum are sufficient to determine the relative probability of ionization, or the relative partial ionization cross sections, to the different ionic states. There are, however, compounds that show significant deviation from this general rule. At best, in cases in which the first PES band lics completely below the incident photon energy one may expect ion yield predictions to be within a factor of two of experimentally determined values. From the aforementioned observed PES trends, and our relative ionization efficiency data at $118 \mathrm{~nm}$, we have observed that the ionization efficiencies using $118 \mathrm{~nm}$ photoionization are high and relatively constant ( $+/-30 \%)$ for many hydrocarbon compounds containing six or more carbon atoms. These compounds have ionization potentials (IPs) a full one to two electron volts below the photon energy $(1182 \AA=10.49 \mathrm{eV})$. The simultaneously-obtained peak ratios can thus be adequate approximations of relative concentration. However for smaller hydrocarbons with ionization potentials lying closer to the single-photon energy and for some larger alkynes, absorption cross sections and ionization efficiencies vary considerably. Consequently, estimation errors for these species are large and individual experimental calibrations are necessary. Even for these species, however, where ion yield at the ionization wavelength is varying sharply with wavelength, ion yields can be estimated as discussed below.

There is quantitative VUV ionization data available in the literature for a limited range of hydrocarbon species which is useful in testing theoretically based calibration strategies. Photoabsorption data and ion yields (Koizumi et al., Person and Nicole and others) allow accurate absolute determination of the photoionization coefficients by simultaneously measuring the absorption cross sections $\left(\sigma_{a}\right)$ and ionization cross sections $\left(\sigma_{i}\right)$ as functions of the incident photon energy. In all cases, photons with an energy from $0-0.3 \mathrm{eV}$ above the first ionization potential give rise to rapidly changing ion yields. For photons above approximately $0.3 \mathrm{eV}$ over the first IP the ion yields for the majority of species reported remain fairly constant, varying by less than $30 \%$ from the first IP to (IP $+2 \mathrm{eV})$. This information can be used to develop a theoreticallybased correlation scheme for estimating single-photon ionization efficiencies.

We have developed a method for correlating VUV photoionization yields, similar to one recently proposed by Koizumi (1991), making use of simultaneously obtained photoabsorption and photoionization cross section of alkanes, alkenes, alcohols and ethers (over the range of $\mathrm{C} 1$ to $\mathrm{C} 6$ ). 
In this paper Koizumi summarizes experimental studies on the decay of superexcited states (states above the ionization potential) of organic molecules. Using a comprehensive set of literature data, the author points out that, in general, in the photoabsorption and decay scheme

$$
A B+h \vee(>I P) \quad-\rightarrow \quad A B^{\prime} \quad-\rightarrow \quad A B^{+}+e^{-} \quad \text { Altoionization }
$$

$\begin{array}{cc}A B^{+}+e^{-} & A+B \\ \text { Direct } & \text { Néltral } \\ \text { Ionization } & \text { Fragmentation }\end{array}$

neutral fragmentation, not autoionization, is the primary channel for decay of the superexcited states. This is the case for all of the alkanes, alkenes, etheres and alcohols examined. This finding is rationalized by noticing that the high energy states in the these species are formed by excitation of an electron in a $\sigma$ bonding molecular orbital, which is likely to leave the molecule in a repulsive part of the highly excited state potential energy curve. Fast dissociation of the neutral excited species then competes effectively with autoionization predominating the decay process.

Due to the fact that most high energy states, $A B^{\prime}$, are Rydberg in nature, the photoabsorption cross section $\sigma_{t}$ in regions of the spectrum above the first ionization potential becomes the sum of the direct photoionization cross sections $\sigma_{\mathrm{i}}$ and the Rydberg excitation cross sections. Since the excited Rydberg electron feels a Columbic field similar to the one in an excited hydrogen atom, Koizumi uses its smoothly decreasing (with increasing principal quantum number) photoabsorption cross sections to model approaching each ionization continuum for each molecular IP.

The separate contributions from each Rydberg series converging to every molecular IP at a given energy are summed with values from the curves fitted using hydrogen atom data:

$$
\begin{aligned}
& \sigma_{t, j}=89.6 \exp \left\{-0.148\left[E-(I P)_{j}+15.43\right]\right\} \\
& \text { for } E>(I P)_{j}-2 e V \\
& \sigma_{t, j}=0 \\
& \quad \text { for } E<(I P)_{j}-2 e V
\end{aligned}
$$




$$
\begin{aligned}
\sigma_{i, j}= & 89.6 \exp \left\{-0.148\left[E-(I P)_{j}+15.43\right]\right\} \\
& \text { for } E>(I P)_{j} \\
\sigma_{t, j}= & 0 \\
& \text { for } E<(I P)_{j}-2 c V
\end{aligned}
$$

Where all j'th ionization potentials are considered.

Figures 2 and 3 present our calculated results for ionization efficiencies of propane and propene when the known IPs are used using the Koizuni treatment.

The Koizumi algorithm can be modified by adding an onset function fitted using an integrated area under an electron band obtained from PES data. This adjustment gives better agreement with experimental ionization efficiencies for compounds where IPs are more broadly spaced (compare for example the curves for propane and propene shown in figures 2 and 3). For alkanes and alkenes this provides a good estimate of photoionization efficiency $(+/-20-30 \%)$. For many alkynes, however, the energy difference between the first and second IP is substantial making simple correlation schemes unusable except for rough estimates (a factor of two or more) of ionization efficiency (alkynes must be calibrated directly).

The above discussion shows the advantages of using VUV photoionization when quantifying concentration clata. Unlike with hot-wire El ionization, where there is always a spread of electron energies, the ionization wavelength is monoenergetic and for many cases theoretically-based estimates of relative photoionization yields can be obtained. Tuning of the ionization wavelength can be used to obtain a degree if species selectivity, to minimize fragmentation probability, and to verify theoretically-based correlation estimates for photoionization efficiency.

\subsection{Discrimination of Isomeric Compounds}

A primary advantage of low intensity VUV photoionization is the relatively soft ionization which it provides. While molecular ion identification is greatly simplified, the structure of the species is not determined unambiguously when using a single ionization wavelength coupled a single mass filter because of the possibility of multiple structures at a single mass which are not selectively ionized. 
Several alternatives have been studied and are discussed below along with suggestions for future work.

\subsubsection{Simultaneous Photoionization and Pulsed Electron Impact Ionization}

For systems where only a few compounds are present in the sampled stream (for example, initial rate studies of pyrolysis and or oxidation of pure hydrocarbon fuels), we have shown that pulsed electron impact ionization simultaneous with photoionization can provide a simple, inexpensive way to analyze both the compound mass and siructure. Two means of using this technique are discussed below.

\section{The UV-LDEI System}

For monitoring multispecies reactions, a substantial benefit is derived when one combines a parentpreserving, nonspecific, and efficient ionization technique with an independently applied fragment generating ionization process for obtaining structural information. For this reason we consider here the consecutive use of VUV laser ionization at $118.2 \mathrm{~nm}(10.49 \mathrm{eV})$ and pulsed electron ionization generated by photoelectron production with $266.0 \mathrm{~nm}(4.66 \mathrm{eV})$ pulsed laser light. Both wavelengths are generated from the same laser by interchanging several optics in and out of the bean path without changing the overall alignment. Coupled to a time-of-flight mass spectrometer (TOF-MS), they serve as complementary techniques to directly observe virtually all of the combustion products from a fast-flow reactor, greatly enhancing both qualitative species monitoring and quantitative analysis of intermediates.

Shown in Figs. 4a and 4b, the third (354.6 nm) and the fourth $(266.0 \mathrm{~nm})$ harmonics of the $\mathrm{Nd}$ :YAG laser fundamental are alternately introduced into the ionization region through the same path. To perform VUV photoionization, $30 \mathrm{~mJ} /$ pulse of the third harmonic of a Nd:YAG fundamental $(1.06 \mu \mathrm{m})$ is tripled in a xenon cell to create $118.2-\mathrm{nm}$ VUV radiation, which is then focused in the ionization region (Fig. 4a). To perform pulsed electron ionization, $3 \mathrm{~mJ} /$ pulse of the fourth harmonic from the same laser was passed through a telescope to obtain an approximately 1.5-2-mm-diam, cylindrical beam which impinges on a small wire $(0.5 \mathrm{~mm}$ diam $)$ positioned approximately at the focal point of the 118.2-nm radiation used in the VUV photoionization runs as shown in Fig. 4b. Ionization occurs in the TOF first acceleration stage, comprised of two electrostatic plates spaced $19 \mathrm{~mm}$ apart and maintained at 1657 and $1031 \mathrm{~V}$. The voltage on the wire was kept below the equipotential surface potential defined by this first acceleration stage (see Fig. 4c) in order to give the electrons a known energy similar to those used in conventional EI-MS. 


\section{The VUV-LDEI System}

Rather than switch wavelengths between ionization sources, the same VUV laser beam can also be used to produce electrons via the photoelectric effect in a fashion similar to that previously discussed for 266- $\mathrm{nm}$ radiation. The ion optics and vacuum system used for this portion of the study, shown in Fig. 5, were different from those used for the UV-LDEI work described above. A frequency-tripled Nd:YAG laser (Quanta Ray DCR-11 or DCR3G system) operating at $10 \mathrm{~Hz}$ is focused through a fused silica lens $(f=125 \mathrm{~mm}$ at $l=266 \mathrm{~nm})$ into a cell containing approximately 26 Torr of xenon which acts as the tripling medium. Approximately $30 \mathrm{~mJ}$ of energy provides optimum VUV signal. The output at $118.2 \mathrm{~nm}$ is focused through a $\mathrm{MgF}_{2}$ lens $(f=150 \mathrm{~mm}$ ) to a focal volume which intersects the molecular beam at a right angle. In practice, using a $\mathrm{MgF}_{2}$ lens, the microjet reactor is mounted $150 \mathrm{~mm}$ from the Xe cell, far from the focal point of the UV beam (at $325 \mathrm{~mm}$ ). The geometry for VUV-LDEI is identical to that normally used for VUV photoionization except for the addition of a wire held at some potential $V_{w}$. Both focused VUV and residual, unfocused UV radiation are transmitted across the ionization volume of the TOF acceleration region bordered by wire-mesh acceleration lenses. The incoming laser beam, the neutral molecular beam, and the wire used for generating electrons are all normal to one another (see Fig. 5). The local pressure at the point of interaction, some 160-200 nozzle diameters downstream from the molecular-beam source, is.estimated to be between $8 \times 10^{-5}$ and $1.2 \times 10^{-4}$ Torr. The wire is inserted approximately at the midpoint of this region $(a=1.6 \mathrm{~cm})$. The potential on this wire is maintained at or below the local potential as clefined by the potentials on the two surrounding ion optics, $V_{1}$ and $V_{2}$. The VUV photons, possessing an energy greater than the work function of the metal (in this case a tantalum wire $0.25 \mathrm{~mm}$ in diameter) strike the surface of the wire, liberating electrons. The work function of the tantalum wire is $4.25 \mathrm{eV}$. As opposed to relatively high-fluence UV laser beams from the same laser, the VUV pulsed radiation is estimated to be converted from the reference UV source with a conversion efficiency no greater than $10^{-5}$. Since the number of electrons generated is directly proportional to the available number of photons having an energy greater than the work function of the electron donating metallic wire, this translates into a significantly lower number of electrons generated when compared to the UV case. However, the advantage resulting from the ability to quickly shift from one ionization method to another and without disturbing the optical path of the radiation is significant. This is especially true when applied to mass spectrometric sampling of high temperature and/or transient reactions in which data acquisition must be performed rapidly. By eliminating the need to manipulate the KDP crystal in the Nd:YAG laser and other optics, and instead either inserting or withdrawing the metallic surface in the paih of the laser beam, switch over from one ionization source to another can be accomplished within seconds. Retuning of the potentials in the acceleration zone of the 
spectrometer is necessary due to the effect of the high potential wire on both the local field and the optimum Wiley-McLauren focusing conditions.

\section{Summary Results from UV or VUV-LDEI}

The determination of the electron energy in both UV and VUV-LDEI is limited by a number of factors, most important of which are the thickness of the wire (contributing to the electron energy spread) and the uncertainty of the wire position with respect to the two electrostatic plates in the first TOF acceleration stage (contributing to the electron energy shift). While the wire thickness introduces a potential energy spread of perhaps $16 \mathrm{eV}$, a position uncertainty of just $\pm 5 \%$ of full range could easily introduce a $\pm 50 \mathrm{eV}$ energy shift. Nevertheless, when the wire voltage is scanned the onset of an EI mass signal consistently appears at an apparent electron energy of approximately $50 \mathrm{eV}$ and remains relatively constant at electron energies of one hundred to several hundred electron volts. Since changes in ionization cross sections of many atoms and molecules are modest in this energy range, the uncertainty in electron energy is less critical. This is confirmed below in comparisons of LDEI fragmentation patterns with those obtained with conventional $50 \mathrm{eV}$ EI sources.

With both VUV and UV-LDEI we were able to obtain good reproducibility for fragmentation spectra as compared to conventional electron impact ionization. One sample fragmentation spectra for methyl acetylene is shown in Fig. 6 along with the fragmentation spectra for conventional 50 $\mathrm{eV}$ EI from the literature. Better reproducibility is obtained if the wire used for generating the electrons is precisely translated in the field. One experimental note is that since the electron energy is a strong function of the position of the wire in the electric field (because of the steep potential gradient) it is important to shield the wire from any high temperature elements (such as the reactor source) in the chamber. Further details about this technique can be found in our paper Boyle et al., 1991.

\subsubsection{Impiovements for Attaining Compound Selectivity Suggested for Future Work}

We have shown that simultaneous use of both VUV photoionization and laser generated electronimpact ionization can simultaneously provide species mass and structural information but only in systems with a small number of measured species and where dissociative relationships between parent and daughter ions are characterizable. This is clearly not the case when conducting flame experiments or high conversion pyrolyses of hydrocarbons, in which a large array of ions of varying structure and molecular weight are simultaneously present. Moreover, many of these species, when fragmented, will decompose into lower mass channels that are already occupied, 
obscuring the data at these mass channels. Deconvolution through "known" fragmentation patterns is computationally ill-conditioned because of the large number of unknowns, and uncertainties in fragmentation pattern functionality.

It would therefore be of significant advantage to provide independent analysis of VUV produced ions of cach mass. One approach involves parent ion selection, dissociation of the chosen parent (either actively or by meta-stable ion decay) and subsequent mass analysis of the daughter ions for a confirming structural analysis. A first mass filter would be used to control the selected mass-tocharge range which is selected to pass. A requirement of this filter to maintain high sensitivity would be high transnission (ca. $90 \%$ or more) throughout the selected range. The TCF-MS as the second mass filter will maximize sensitivity, since the dispersion of daughter ions is longitudinal and not lateral. This allows a much greater number of ions of all possible masses to reach the detector, rather than just ions of a single mass which satisfy the temporal scanning function of some electric or magnetic field.

A schematic diagram of a proposed TOF-MS system optimized for VUV photoionization is shown in Fig. 7. This system would offer high sensitivity (at least sub-ppm with seconds averaging), and mass resolution (single mass resolution up to at least $1000 \mathrm{amu}$ ) and the ability to distinguish isomeric components. The VUV-MS system consists of a tunable laser-based VUV photoionization source and a time-of-flight mass filter, separated by a two sector (E $\times B$ ) mass spectrometer and a means for dissociation of selected ions. In practice, this device could be run manually and, in the absence of a dissociation media, set to transmit molecules of all mass-tocharge values simultaneously for complete parent ion data, much as the single TOF instrument we are currently using. Alternately, the window of mass-to-charge values accepted by the mass filter could be narrowed to a single mass range, allowing the dissociation conditions to be optimized for a given mass analysis. Data collection and analysis will be through either pulse counting or analog circuitry, depending on signal amplitudes and repetitiveness. The necessary signal processing electronics are already in place in our laboratory. In addition, the current system also includes a separate mass analyzer for cross calibration of ion yields.

In summary, a VUV photoionization-MS system can be configured to allow sensitive mass detection in a complex mixture while also providing structural information. Sensitivity of the technique will be improved by better matching of the neutral-producing and photon-producing duty cycles and use of ion counting techniques. Selectivity for structural studies can be provided using simultaneous pulsed El (low conversion data only), tandem mass filters (as shown in Figure 7), 
variable wavelength VUV photoionization, or by a number of other spectroscopies in the sampled product beam.

\subsection{Irydrocarbon Pyrolysis and Oxidation Combustion}

Details of two hydrocarbon reaction studies are discussed below to illustrate the utility of the VUVMS technique (for coal processing analysis). Both examples illustrated demonstrate the advantages of VUV-MS for providing mechanistic insight in complex hydrocarbon reaction systems.

\subsubsection{Higher Ilydrocarbon Production During Allene Pyrolysis}

Summary: Pyrolysis of allene in a microjet reactor at millisecond to second reaction times over a temperature range from 500 to $1700 \mathrm{~K}$ was used to study higher hydrocarbon growth processes from $\mathrm{C}_{3}$ hydrocarbon species. Species detection of both stable and labile product masses was carried out using VUV photoionization mass spectrometry. The first product observed with increasing temperature at ms time scales was mass 80 followed at somewhat higher temperatures by smaller than parent-mass pyrolysis products. Low temperature benzene formation likely occurs through the molecular channel consisting of allene dimerization to a mixture of dimethylenecyclobutane isomers (DMCB) and subsequent isomerization and hydrogen loss to form benzene. Mass 79 is observed at concentrations up to half the mass 80 concentration prior to mass 78 detection. The allene dimerization step is fast enough to account for the initial rate of mass 78 production observed prior to significant allene decomposition. The 1,2 DMCB also reacts with allene to form a trimer at mass 120 . The appearance of other high molecular weight products at masses 92, 116, 114 and 158 is also observed before allene decomposition occurs at greater than several percent levels. At higher temperatures and longer residence times, however, the benzene production rate shows a change in formation mechanism through a change in the apparent activation energy observed. At temperatures greater than $1580 \mathrm{~K}$ and ms time scales, mass 78 production was consistent with formation through propargyl radical recombination. Higher hydrocarbon growth to multiple ring compounds was also observed at longer residence times with growth initially consistent with acetylene addition to PAH radicals followed by reactive dimerization of small PAH compounds with the first dimers observed in the 400-600 amu range.

\section{Background}

It has become increasingly apparent in the past several years that $C_{3}$ routes for soot formation in flames can be important under some if not many conditions. In several recent studies of fuel pyrolysis and combustion where both stable and labile product measurements were made (e. g. Westmoreland et al, Boyle and Pfefferle), $\mathrm{C}_{4} / \mathrm{C}_{2}$ growth mechanisms were determined to be not fast enough to account for observed benzene production for some of the fuels used, especially 
those of $\mathrm{C}_{2}$ and $\mathrm{C}_{3}$ hydrocarbons. Westmoreland et al. have recently made a $Q R R K$ reaction pathway analysis of propargyl radical $\left(\mathrm{C}_{3} \mathrm{H}_{3}(\mathrm{P})\right)$ dimerization and concluded that this route could in fact be fast enough to account for observed benzene production rates in several test flames including acetylene and ethylene. This mechanism had been proposed by Kern and Wu to explain benzene production in pyrolysis of allene. An analysis of benzene production from propargyl radical has also been studied by Stein and coworkers and found to be a likely source of benzene production at flame conditions.

At low temperatures, however, the molecular channel for benzene formation can predominate (Blomquist and Verdol, Meinert and Hurd). In early studies of the gas phase pyrolysis of allene at $800-900 \mathrm{~K}$, Blomquist and Verdol observed dimethylenecyclobutane isomers as the primary reaction products. The 1,2-dimethylenecyclobutane (1,2 DMCB) isomer was produced at high yields by passing allene over quartz at $800 \mathrm{~K}$ with a $6 \mathrm{~s}$ mean residence time. Quantitative data was not obtained because of the high rate of polymerization of allene and its dimer.

In addition, surface effects which are probably significant in this system due to the high exothermicity of the dimerization reaction were not accounted for. In the current study mass 80 (likely formed through allene dimerization) was the first product detected at low temperatures and residence times. At higher temperatures mass 78 is more predominant than mass 80 and two other $\mathrm{C}_{3}$ channels have been proposed to play important roles in the formation of benzene. These are given in overall form below:

$2 \mathrm{C}_{3} \mathrm{H}_{3}(\mathrm{P}) \rightarrow \mathrm{C}_{6} \mathrm{H}_{6}$ (linear) $\rightarrow$ benzene

$$
\mathrm{C}_{3} \mathrm{H}_{3}+\mathrm{C}_{3} \mathrm{H}_{4}(\mathrm{~A}) \rightarrow \mathrm{C}_{6} \mathrm{H}_{6} \text { (benzene) }+\mathrm{H}
$$

Both of these mechanisms were tested with the concentration dependence and temperature dependence observed. $\mathrm{C}_{4} / \mathrm{C}_{2}$ mechanisms were also initially tested but found to predict benzene production rates that were too low, especially as temperature increases in the 1550-1650 range where allene decomposition becomes fast and total allene conversion is $>90 \%$ at $2 \mathrm{~ms}$.

This study was also expanded to the pyrolysis of allene at longer (s) residence times to explore the pathway from PAH/high molecular weight hydrocarbon species to soot precursors, which is an important unresolved issue. Prior researchers have illustrated (e.g. Calcote) that acetylene addition to condensed polyaromatic hydrocarbons is not alone fast enough to create soot masses in the ms time frame observed in flames. In addition, the $\mathrm{H} / \mathrm{C}$ ratio of "soot nuclei" produced solely by the 
acetylene addition mechanism followed by coagulation of species of mass $1000 \mathrm{amu}$ and higher is too low to be consistent with the relatively high $\mathrm{H} / \mathrm{C}$ ratio measurements $(0.5)$ of young soot particles (Wagner). Some intriguing new data (Santoro, D'Alessio) suggests formation of a soot precursor class that is more aliphatic in nature than PAH. Early work by Homann and Wagner also found evidence for a class of reactive hydrocarbons containing more hydrogen than PAH. These factors suggest that some mechanism in addition to acetylene addition to PAH radicals is important in forming species that become the precursors for the initial soot nuclei. One possible mechanism involves reaction of large benzylic type radicals or diradicals with other stable PAH. The long residence time allene pyrolysis product profiles provide evidence for reactive dimerization of small PAH species (128 to $408 \mathrm{amu}$ ) leading to rapid growth in masses in the 408 to $662 \mathrm{amu}$ range and explores possible mechanisms for such a dimerization process.

\section{Experimental Procedure}

Allene $\left(\mathrm{C}_{4} \mathrm{H}_{3}(\mathrm{~A})\right)$ pyrolysis was carried out in a microjet reactor source, described in detail above (Pfefferle et al., Boyle). This is a miniature fast-flow reactor coupled directly to a sonic nozzle with a volume of approximately $3.2 \times 10^{-9} \mathrm{~m}^{3}$. Reactants (pure allene or allene/ $\mathrm{O}_{2}$ in this study) are introduced to the pyrolysis zone through the center-most hole in the inner alumina tube $(0.4$ $\mathrm{mm}$ ID) and expanded into the reaction chamber. The reaction zone is resistively heated and temperature within the reactor zone has been calibrated using thermocouples. Thermocouples were not used continuously during experiments due to the catalytic oxidation/pyrolysis observed on the platinum/rhodium wires. Pressure within the microjet reactor was maintained at $600 \pm 20$ torr. Under the stated operating conditions, wall reactions do not significantly affect product distributions, and collisions with the wall are much less frequent than molecule-molecule collisions. The variation in reaction product distribution with absolute pressure does not indicate significant contribution of the surface until pressure is decreased by a factor of 10 . A test of wall inertness was made through pyrolysis of cyclohexane in both alumina and quartz tubes; this showed no evidence of differences in the mass spectra at temperatures from $300-1600 \mathrm{~K}$. Our analysis of this reactor (including modeling of $\mathrm{CO} / \mathrm{CO}_{2}$ production in combustion mode, and ion residence time studies) suggests that for short (ns) residence time operation it can be modeled as a well mixed reactor. In addition, under the low conversion reaction conditions we use in this study the differential reactor assumption can be used in the analysis of the initial reaction pathways.

\section{Experimental Results and Discussion}

Figure 8 shows the mass spectra from allene pyrolysis at increasing temperatures including $1320 \mathrm{~K}, 1360 \mathrm{~K}$ and $1515 \mathrm{~K}$ and $1660 \mathrm{~K}$ at $2 \mathrm{~ms}$ mean residence time. These spectra are raw signal averaged data from a single run with no calibration corrections applied and the mass 40 peak is off- 
scale for all except the highest temperature. As temperature was increased from $300 \mathrm{~K}$ to $1200 \mathrm{~K}$, the first reaction product observed was mass 80 followed at slightly higher temperatures by smaller reaction products such as mass 28 (ethylene) and mass $52\left(\mathrm{C}_{4} \mathrm{H}_{4}\right)$. Flow reactor results also indicate methane and hydrogen in significant quantities, although those products were not directly measured in this experimental configuration due to their high ionization potentials (methane was indirectly meastirable through its fragmentation product methyl radical). Mass $52\left(\mathrm{C}_{4} \mathrm{H}_{4}\right)$ is the $\mathrm{C}_{4}$ species with the highest concentration up to $1450 \mathrm{~K}$ and is observed to increase coincident with ethylene. These species are observed prior to rapid allene decomposition, and are consistent with allene dimer formation to dimethylenecyclobutane (DMCB) isomers followed by decomposition through the reaction below:

$$
D M C B \rightarrow \mathrm{C}_{4} \mathrm{H}_{4}+\mathrm{C}_{2} \mathrm{H}_{4}
$$

Starting at $1320 \mathrm{~K}$ a range of high mass species in addition to mass 80 including masses 79,92 , 106 and 120.were observed to reach significant steady state concentrations prior to significant production of mass 78 . In fact in this system, benzene production was not necessarily the rate limiting step to higher hydrocarbon production. It should also be noted that the conceritration of mass 92 increases to observable levels at low temperatures prior to significant mass 78 production (see Fig. 8 and Table 1). A possible low temperature pathway is the reaction of propargyl radical with vinyl acetylene:

$$
\mathrm{C}_{4} \mathrm{H}_{4}+\mathrm{C}_{3} \mathrm{H}_{3} \rightarrow \mathrm{C}_{7} \mathrm{H}_{7} \rightarrow \rightarrow \text { toluene }
$$

The reverse of this reaction has been observed to be an important lower temperature channel in the decomposition of benzyl radical. At low temperatures $(\mathrm{T}<1200 \mathrm{~K})$ in their toluene pyrolysis study, Pamidimukkala et al. determined the major pyrolysis products to be $\mathrm{C}_{7} \mathrm{H}_{7}$ followed by $\mathrm{C}_{5} \mathrm{H}_{5}, \mathrm{C}_{5} \mathrm{H}_{3}, \mathrm{C}_{4} \mathrm{H}_{4}$ and $\mathrm{C}_{4} \mathrm{H}_{2}$. In subsequent tests with $\mathrm{C}_{7} \mathrm{H}_{7} \mathrm{Cl}$ these investigators noted that the $<\mathrm{C}_{7}$ products were secondary cracking products from $\mathrm{C}_{7} \mathrm{H}_{7}$ not $\mathrm{C}_{7} \mathrm{H}_{8}$.

Mass 120 detected at temperatures as low as $1280 \mathrm{~K}$ was likely formed through reaction of mass 80 with allene as noted in early work on the thermal polymerization of allene. Mass 120 and 160, which are possible allene trimers and tetramers, were not detected in our studies of ethylacetylene pyrolysis (Pfefferle et al., Boyle) or in our studies of methyl acetylene pyrolysis (Pfefferle et al.) (at temperatures and reaction times where isomerization is not equilibrated) but are present at significant ievels in this study. This is another indication of allene dimer formation in this system and the low temperature reaction conditions. 
A mechanism for allene dimer and trimer formation was reported by Weinstein and Fenselau and is given below:

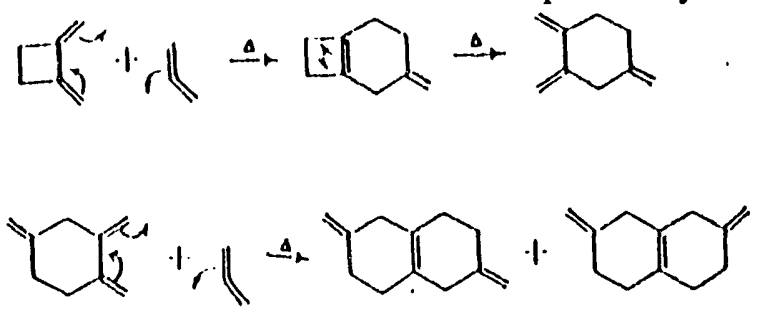

At higher temperatures and allene conversions, these species are converted through thermal decomposition and reactions with small hydrocarbon radicals.

It is instructive to compare the results from the lower temperature end $(1100-1515 \mathrm{~K})$ of this study with those of Hidaka et al. for dilute allene and propyne pyrolysis behind a reflected shock wave at $\sim 2 \mathrm{~ms}$ residence time and $\mathrm{T}=1200-1500 \mathrm{~K}$ at somewhat higher pressures $(1.7-2.5 \mathrm{~atm})$ than this study $(1.1 \mathrm{~atm})$. Even though the allene/propyne isomerization is relatively fast for these conditions, it was not equilibrated and these researchers observed considerable differences in both product profiles and total $\mathrm{C}_{3} \mathrm{H}_{4}$ conversion between runs starting with either allene or propyne. At $1400 \mathrm{~K}$ for dilute (4\% in $\mathrm{Ar}$ ) propyne/allene pyrolysis they reported $>45 \%$ total $\mathrm{C}_{3} \mathrm{H}_{4}$ conversion for allene and $>30 \%$ conversion for propyne (both at $\sim 2 \mathrm{~ms}$ ). The total $\mathrm{C}_{3} \mathrm{H}_{4}$ conversion reported for allene is over twice that observed in this study (for the same temperature) at higher allene concentration but about half the total pressure. During allene pyrolysis more $\mathrm{C}_{6} \mathrm{H}_{6}$ was formed than cluring propyne pyrolysis especially between $1350-1500 \mathrm{~K}$, peaking at $1450 \mathrm{~K}$ and then dropping off sharply at higher temperatures. The other differences included more $\mathrm{C}_{3} \mathrm{H}_{6}$ and $\mathrm{C}_{4} \mathrm{H}_{4}$ for allene pyrolysis and considerably more $\mathrm{C}_{2} \mathrm{H}_{4}$ for allene pyrolysis, especially at the lower temperatures, from $1250 \mathrm{~K}-1350 \mathrm{~K}$. Ethylene drop off for the allene case was coincident with the fall-off in net benzene production observed. Dimethylenecyclobutane (DMCB) was inferred to be formed as the initial product at low temperature by comparison of $225 \mathrm{~nm}$ absorption measurements with gas chromatographic measurements of allene and propyne. This is consistent with the observations of the current study for mass 80 appearance prior to significant allene decomposition. The greater production of $\mathrm{C}_{2} \mathrm{H}_{4}$ and $\mathrm{C}_{4} \mathrm{H}_{4}$ observed during allene pyrolysis, was likely a result of the decomposition of $\mathrm{DMCB}$ isomers ( $\mathrm{R} 3$ above) and is also consistent with the current study. Although no $>\mathrm{C}_{6}$ hydrocarbons were reported in the study of Hidaka et al., tars were noted on the tube wall at $\mathrm{T}>1450 \mathrm{~K}$, which is also consistent with the high mass measurements in the current study at relatively low temperatures.

An overview of the approximate concentrations of higher hydrocarbons detected at $2 \mathrm{~ms}$ reaction times at temperatures ranging from $1280-1580 \mathrm{~K}$ are given in Table 1 . This data represents 
aver ges for several different runs at each temperature and includes normalization for estimated relative detection efficiency. Below $1450 \mathrm{~K}$, high mass peaks are located at $79,80,92,106$ and 120. As temperature was increased above $1450 \mathrm{~K}$ a broad range of hydrocarbons with 6 or more carbons were detected including mass 78 with the largest contributions initially coming from masses $92,94,106,116,120,144$ and 158 . At $1450 \mathrm{~K}$ the pyrolytic decomposition of the total $\mathrm{C}_{3} \mathrm{H}_{4}$ component (allene + propyne) becomes fast, and $\mathrm{C}_{4}$ and $\mathrm{C}_{5}$ radicals reach appreciable concentrations (see Table 1). At this point radical growth processes for higher hydrocarbon growth are expected to dominate over the molecular growth processes observed at low temperatures. Processes governing benzene production in the two growth regimes are discussed below.

\section{Low Temperature Benzene Formation Mechanism}

The data from both this study and that of Hidaka et al. suggests that at low temperatures $(<1400 \mathrm{~K})$ and ms time scales the molecular channel for $\mathrm{C}_{6} \mathrm{H}_{6}$ formation through dimerization to a DMCB isomer is may be important low temperature route. As noted above, Hidaka et al. observed an excess of $\mathrm{C}_{6} \mathrm{H}_{6}$ from pyrolysis of allene $\left(\mathrm{C}_{3} \mathrm{H}_{4}(\mathrm{~A})\right)$ over propyne $\left(\mathrm{C}_{3} \mathrm{H}_{4}(\mathrm{P})\right)$ at similar reaction times to this study $(1-3 \mathrm{~ms})$ and temperatures $(1200-1500 \mathrm{~K})$. At temperatures $>1250 \mathrm{~K}$ benzene was confimed by GC measurements. This is consistent with our studies showing how the molecular channel can contribute to $\mathrm{C}_{6} \mathrm{H}_{6}$ production even at moderate temperatures with respect to allene/propyne isomerization. This view is supported by the observation of more $\mathrm{C}_{2} \mathrm{H}_{4}$ and $\mathrm{C}_{4} \mathrm{H}_{4}$ from the allene pyrolysis than from propyne pyrolysis even though isomerization between allene and propyne is relatively fast at these conditions but not equilibrated on the 1-3 msec time scale. As pointed out by Kiefer and coworkers, this suggests a contribution to benzene production from the molecular channel rather than through $\mathrm{C}_{3} \mathrm{H}_{4}(\mathrm{~A})+\mathrm{C}_{3} \mathrm{H}_{3}$ which does not produce the correct concentration dependence or as strong a negative temperature dependence as observed.

A first step to test the viability of the possible molecular channel to benzene was to estimate rates for allene dimerization to DMCB isomers and compare the rate with the experimentally observed rate for mass 80 production at low conversion. The themodynamic parameters were estimated using the THERM (Ritter and Bozzelli) program and checked with experimental values where available. Equilibrium constants for the reactions 2 Allene $=1,2-\mathrm{DMCB}$, and 2 Allene = 1,3$\mathrm{DMCB}$ were computed and found to be greater than one up to about $1400 \mathrm{~K}$. At temperatures below $800 \mathrm{~K}$ the primary isomer predicted during early high concentration allene pyrolysis studies (Blomquist and Verdol, Meinert and Hurd) was 1,2-DMCB. At higher temperatures, however, the 1,3-isomer becomes an important product $(>10 \%)$. 
The rate constant for allene dimerization to 1,2-DMCB was estimated using transition state theory, analogy to similar reaction and limited experimental data. The dimerization rate of allene to 1,2DMCB is pressure dependent and highly exothermic (approximately $40 \mathrm{Kcal} / \mathrm{mol}$ ). Table 2 shows the energetics of the reaction and the prediction for the dimerization rate at two temperatures using a QRRK analysis with an argon bath gas at $1 \mathrm{~atm}$. The activated complex for the rate limiting configuration was estimated to be approximately $15 \mathrm{Kcal} / \mathrm{mol}$ above the reactants by Gajewski and Shih. The reverse rate was calculated from equilibrium, and the rate was extrapolated to our conditions through QRRK analysis using argon as the bath gas. Experimental rate data available for dimerization to $1,2-\mathrm{DMCB}$, along with rate data of similar reactions were used to check our estimate (e.g. pyrolysis of methylenecyclobutane to allene and ethylene). The estimated rate reported on Table 2 for 700 and $800 \mathrm{~K}$ is close to the higher experimental value reported in the earlier literature(Blomquist and Verdol, Meinert and Hurd, and references therein) shown on Table 3. These early mea: urements of allene dimerization did not account for tar formation which depleted the dimer concentration, and surface effects which accelerate the rate could also have been present. The rate constant determined in this study at the low temperature end $(800 \mathrm{~K})$ is also close to that predicted by our estimate but it should be recognized that the error bar on this estimate is likely at least $\pm 50 \%$, and the error bar on the experimental concentration estimate is about the same considering that other $\mathrm{C}_{6} \mathrm{H}_{8}$ isomers likely present with varying detection efficiencies.

The estimates for allene dimerization rates discussed above are consistent with the our experimental measurement's of mass 80 production, this does not, however mean that early mass 78 production observed takes place through the mass 80 intermediate. The most probable thermal rearrangements of 1,2-DMCB, do not lead to benzene intermediates. Rupture of the carbon bond between the two methylene groups does produce species that could subsequently react to form benzene at appreciable net rates but this would be a relatively high activation energy reaction. One other possible route which is consistent with the data would be relatively fast conversion of 1,3-DCMB to methylene cyclopentene and then 1,3-cyclohexadiene, followed by formation of the cyclohexadienyl radical at mass 79 . This mechanism would be consistent with the large mass 79 peak observed prior to mass 78 appearance. Benson and Shaw proposed a multistep mechanism for conversion of 1,3-cyclohexadiene to benzene initiated by:

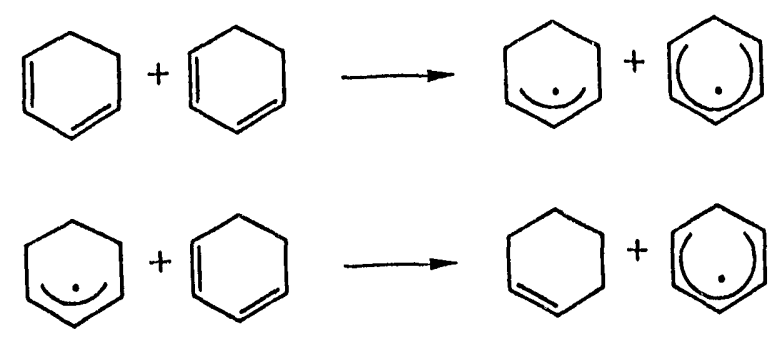


Followed by benzene formation through:

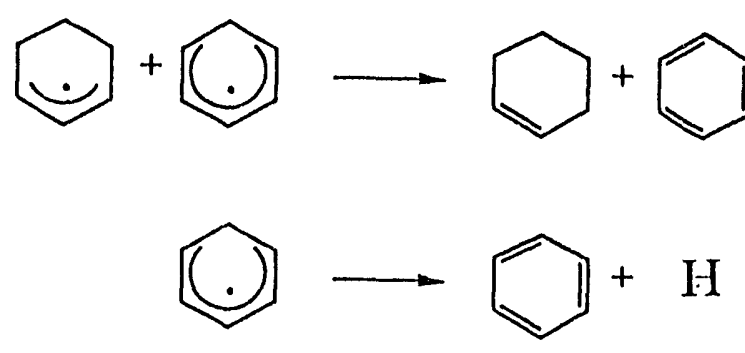

This mechanism provides one explanation for the large steady state mass 79 concentration observed in our experiments. Mass 82 is also observed in our experiments at relatively low $(1360 \mathrm{~K})$ temperatures. Figure 9 shows relative concentrations of selected species as a function of temperature ( $2 \mathrm{~ms})$. Mass 79 discussed above parallels mass 80 production until $1450 \mathrm{~K}$ suggesting that one is directly produced from the other as suggesiëd above. Direct pyrolysis studies of 1,2- and 1,3-DMCB must be carried out to clarify whether a direct route to benzene from these precursors can play a role in low temperature benzene production during allene pyrolysis.

\section{High Temperature Benzene Formation}

At higher temperatures in the ms residence time regime, the benzene formation mechanism changes. This can be observed from the change in the temperature dependence of the benzene formation (see Figure 9). The change in slope corresponds to the temperature rate at which allene conversion is also increasing rapidly from $<1 \%$ (at $1380 \mathrm{~K}$ ) to $>15 \%(1400 \mathrm{~K}$ ) at $2 \mathrm{~ms}$ 'and where the main conversion route changes from dimerization to decomposition as noted by the marked increase of product masses indicative of radical growth processes. It should be noted that at $1450 \mathrm{~K}$ production of $>78$ amu species is significant so the actual mass 78 production rate is greater than directly observed. An inilial survey of possible mechanisms and the concentrations of possible precursors observed in this study showed that only the two C3 channels (R1 and R2) could account for the order of magnitude of mass 78 production in the $\mathrm{T}>1515 \mathrm{~K}$ range. Although we do not have an estimate for absolute $\mathrm{C}_{3} \mathrm{H}_{3}$ concentration in these experiments, the concentration is observed to increase sharply with temperature from 1400 to $1600 \mathrm{~K}$ coincident with the mass 78 curve. A significant change in the net rate of formation of benzene and other $>$ 78 amu species does not occur until after $\mathrm{C}_{3} \mathrm{H}_{3}$ concentration rises significantly (i.e. mass 78 production rate is more closely a function of $\left[\mathrm{C}_{3} \mathrm{H}_{3}\right]^{2}$ than $\left.\left[\mathrm{C}_{3} \mathrm{H}_{4}(\mathrm{~A})\right]\left[\mathrm{C}_{3} \mathrm{H}_{3}\right]\right)$. Considering equilibrium for the reverse of the reaction $\mathrm{C}_{3} \mathrm{H}_{4}(\mathrm{~A})+\mathrm{C}_{3} \mathrm{H}_{3} \rightarrow \mathrm{C}_{6} \mathrm{H}_{6}+\mathrm{H}$, this reaction could not produce a large positive increase in benzene production with temperature over the range from 1400 $-1600 \mathrm{~K}$.

Hidaka and coworkers observed more benzene formation from allene pyrolysis than from propyne pyrolysis, suggesting that the reaction $\mathrm{C}_{3} \mathrm{H}_{4}(\mathrm{~A})+\mathrm{C}_{3} \mathrm{H}_{3} \rightarrow \mathrm{C}_{6} \mathrm{H}_{6}+\mathrm{H}$ could be responsible for this difference. As we have discussed above, however, the observed difference for $\mathrm{C}_{3} \mathrm{H}_{4}(\mathrm{~A})$ pyrolysis 
is more likely due to a residual contribution to mass 78 production from the molecular channel at temperatures below $1450 \mathrm{~K}$ at ms time scales as discussed above.

\section{Higher Hydrocarbon Formation at Long Residence Times}

The allene pyrolysis experiments were repeated in the temperature range from $1100-1500 \mathrm{~K}$ at long (s) residence times to investigate mechanisms leading to large hydrocarbon and PAH production. Figure 10 illustrates the product spectra in the mass range 0-700 amu for neat allene pyrolysis at $1495 \mathrm{~K}, 12$ seconds mean residence time and $915 \pm 20$ Torr. The data in figures $10-13$ has been averaged over 100 laser shots but has not been normalized for efficiency of individual mass detection. At these conditions over $99 \%$ of the allene has been converted to products with most of the carbon mass present as higher molecular weight species. This residence time/temperature snapshot is interesting because it shows the initial growth pattern of species in the mass range > 400 amu. Figures 10-12 show expanded sections from this spectra at several high mass ranges to highlight the different growth mechanisms observed.

Figure 11 shows the mass range 152-300 amu at five times the magnification of Figure 10. This figure shows clearly that the growth to higher hydrocarbons in this mass range is consistent with the acetylene growth mechanism. The mass numbers correspond to stoichiometries of structures that are consistent with $\mathrm{C} 6$ and $\mathrm{C} 5$ rings in conciensed configuration. The observed stoichiometries are consistent with results by Wagner and coworkers, who in detailed LC/MS studies of PAH production in this mass range in flames over a range of pressures showed no evidence of significant concentrations of PAH with side chains.

Figure 12 shows detail of the product distribution in the mass range 302-408 in which peaks consistent with growth by sequential acetylene additions are also present, however more filling in of the spectrum is observed to account for a greater ringe of stable structures due to the different degree of ring packing arrangements possible for a given number of carbon atoms. A striking difference in the spectrum of this mass range is the approximately equal concentrations of major peaks increasing in concentration at mass 408 , illustrating the possibility of another growth mechanism that becomes more important at higher masses. An acetylene polymerization mechanism in the absence of an equilibrium constraint would result in an approximate exponential decay of product concentrations on the high mass side of the species with the maximum concentration. This is clearly not the case in the mid to high 300 amu mass range (figure 12) but is observed past the PAH maximum (at 228 amu) in the mass range from 228 amu to 324 amu 
(figures 11 and 12). At mass 408 a distinct change in the spectrum is observed. Mass 408 abundance is greater than any species in the 300 amu range, and fewer side peaks are present.

Figure 13 shows a ten times enlargement of the mass range 408-662. In this mass range significantly fewer product masses are observed. Each peak is distinct and the spacing is consistent with these species being products of dimerization of predominant peaks in the low mass PAH spectra. This is illustrated on Table 3 which shows dimer possibilities $\mathrm{A} 1+\mathrm{A} 2=\mathrm{Dimer}-\mathrm{H}_{2}$. What is apparent is that the major peaks can be predicted by reaction of masses which have isomer structures with saturated/partially satturated carbon/carbon bonds. It has been noted in previous studies that hydrogenation of $\mathrm{C}$ bonds in PAH can take place appreciably at high combustion temperatures ( $\mathrm{T}>165(\mathrm{~K}, \mathrm{~ms}$ residence times). These types of compounds have been detected by $\mathrm{LC}$ in roughly equal concentrat:ons to condensed PAH structures during the pyrolysis and oxidative pyrolysis of a wide variety of aromatic compounds as well as n-decane (Bruinsma and Moulijn). The observed product distribution is also consistent with pre-sooting products observed in a study of soot formation from coal (Sarofim).

The (yuestion of whether this data can be extrapolated to examine the possible role of the reactive dimerization in an actual flame enviromment can be addressed roughly by comparing the growth rate of species in the $20(5-408$ amu range (assumed produced by acetylene addition to PAH radicals) and the $\triangle(08-662$ amu range (assumed produced by the irreversible reaction of PAH species). The rates for each jrocess are

$$
\begin{aligned}
& \left.\mathrm{r}_{\text {acclylenc addition }} \quad=\quad \mathrm{k}_{\mathrm{a}} l \mathrm{C} 2 \mathrm{H} 2\right][\mathrm{PAH} \cdot] \text { and } \\
& \mathrm{r}_{\text {cimcrization }}=\mathrm{k}_{\mathrm{d}}[\mathrm{PAH}][\mathrm{PAH} \cdot] \text {. }
\end{aligned}
$$

Therefore, in order to extrapolate the results of this study to flame conditions, observed dimerization rates must be normalized by the ratio of

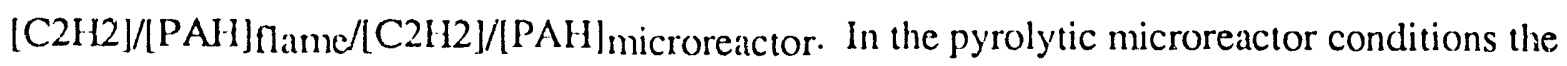
PAH concentration is significantly higher than in a flame. For the conditions of this study the ratio

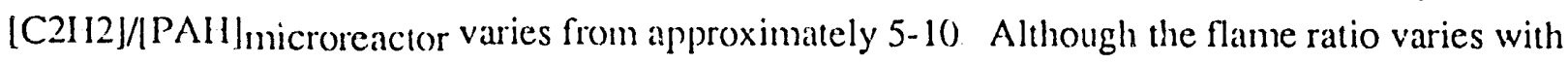
conditions (fuel, local equivalence ratio and $\mathrm{T}$ ), pre-sooting values for the $[\mathrm{C} 2 \mathrm{H} 2] /[\mathrm{PAH}] \mathrm{flame}$ ratio is estimated at approximately 100 from concentration profiles reported in the literature (Blonquist and Verdol, Lam et al., Longwell, Glassman). Therefore to compare the relative importance of the dimerization reaction mechanism in the microreactor to actual flame conditions the observed rate would have to be divided by a factor of $10-20$. It should be noted that as discussed earlier detection sensitivity is dropping with mass in the dimerization mass range and therefore this process appears to be less dominant in Figures 12 and 13 than it actually is. 
Apparent growth rates of species in the 408-662 amu range are several times as fast as those in 300 - 408 amu range from the same precursor pool, with 3-12 times the carbons added per reaction for monomers in this mass range (carbon addition factor increases with monomer size). Therefore in this size range, the rate growth from a given monomer to twice its mass by the dimerization growth mechanism would be competitive with that by acetylene addition to PAH radicals, and this advantage would increase with monomer size. Uncertainties in this extrapolation were also evaluated. First, the PAH radicals in both mechanisms are not necessarily the same structure (phenylic type or benzylic type) and have different thermal stability. Benzyl radicals are more stable in this temperature range than phenyl radicals. The relative concentrations of these radical types, however, are also affected by their precursor concentrations and kinetic barriers for formation from the precursor. These factors are also affected by the presence of oxygen. Our results for pyrolysis of benzene and toluene in the presence of oxygen suggest that both abundance of small side chain substituted PAH and dimers that are consistent with benzylic radical addition to an aromatic ring structure are more, not less, predominant in the presence of oxygen.

We have shown above that a reactive dimerization growth mechanism may be competitive with other higher hydrocarbon growth mechanisms in a flame for species in the mass range $>400$ amu. What implications does this have for formation of soot precursors in flames? Is it more likely that species formed through this growth mechanism lead to growth of soot muclei? Two considerations that may help answer these questions are the thermal stability of these species and their $\mathrm{H} / \mathrm{C}$ ratio. Thermal stability is not directly addressable because we do not know the structure of the proposed dimers. It is clear however that species with multiple bridged or ladder structure would have high thermal stability. H/C ratio as a function of growth mechanism can be directly addressed. The possible stoichiometries of species produced from either the acetylene growth mechanism or the reactive dimerization mechanism for products in the 408-662 mass range are illustrated on figure 13 for each major peak. It should be noted that the stoichiometry of species produced through the acetylene growth mechanism alieady have an $\mathrm{H} / \mathrm{C}$ ratio less than that of young soot $(0.26-0.36)$ whereas the products postulated by a reactive dimerizattion mechanism have a $H / C$ ratio consistent with a soot precursor (0.49-0.75). Note that the highest molecular weight shown in Fig. 13 (662 amu) would have an $1 / / C$ ratio of 0.26 if grown by the acetylene growth mechanism and 0.49 if grown by a dimerization mechanism. Therefore, if formed by either growth mechanism the 662 amu species would hive an I/C ratio at or lower than that of young soot. In addition, even old soot has a fairly high , () 25-(0.2) $\mathrm{H} / \mathrm{C}$ ratic. These observations reinforce the idea that a mechanism for soot precursor formation other than sriely acetylene addition to $\mathrm{PAH}$ radicals must be important at least in the PAH size range $>300$ amu. This has been stated earlicr by Wagner (Santoro), "In order for the fuel molecules to evolve to snot, there must take place a (chemical!) condensation of 
species with the right hydrogen content or a (chemical!) condensation of species with a higher hydrogen content and consecutive dehydrogenation or a combination of these two pathways." We have shown that chemical dimerization can take place during the pyrolysis of allene and that this mechanism likely has relevance for flame conditions.

\section{Conclusion of the Allene Pyrolysis Study}

A microjet reactor coupled to a vacuum-UV photoionization time-of-flight mass spectrometer was used to study the formation of higher hydrocarbons during the pyrolysis of allene. By use of this technique, a progression of pyrolysis product spectra including hydrocarbon radicals were obtained as a function of temperature at millisecond to second reaction times. Mass 80 was the first product species observed as temperature was increased from $300 \mathrm{~K}-1200 \mathrm{~K}$ at ms reaction times. This is consistent with early flow reactor studies of allene pjrolysis where allene dimerization leading to the formation of predominantly the 1,2 isomer of dimethylenecyclobutane was observed. An analysis made of a proposed mechanistic pathway for allene dimerization to DMCB isomers and possible mechanisms for subsequent conversion to benzene showed a molecular growth mechanism is consistent with the low temperature mass 78 observed in this and previous studies.

At higher temperatures the mass 78 (benzene) production was observed to be consistent with formation predominantly through $\mathrm{C}_{3} \mathrm{I}_{3}$ recombination. This is observed both through the concentration dependence and the temperature at which the benzene formation rate increases dramatically.

At long (second) residence times and low temperatures (1495K), the formation of multiple ring hydrocarbons was observed. In the mass range from 150 to 350 amu growth was observed to be consistent with successive acetylene addition to PAH radicals. At higher molecular weights, however, a second competitive mechanism was observed consisting of reactive dimerization of small PAH/PAH radicals to form dimers first clearly distinguishable in the mass range $>400$ amu. This mechanism was estimated to be important at flame conditions and provides one explanation for the high $\mathrm{H} / \mathrm{C}$ ratio observed in young soot.

\subsubsection{Benzene Oxidation and the Oxidative Pyrolysis of Benzene}

A second example of the application of VUV-MS that will be described in detail is the oxidation and oxidative pyrolysis of benzene. Aside from the relevance of this system to molecular growth in soot inception processes, it can be viewed as a test case for some complexities expected in the sampling of coal pyrolysis and combustion. 


\section{Procedure}

Premixed gases were delivered to the microjet reactor via a mass flow controller (Brooks Instruments, Hatsfield, PA). Before its use in mixture preparation, benzene (Baker Analyzed, Reagent Grade) was outgassed by three consecutive freeze-pump-thaw cycles. During all the experiments reported here, the analysis chamber pressure was maintained below $5 \times 10^{-5}$ torr and in the TOF tube below $2 \times 10^{-6}$ torr. All results presented here are for a 0.340 mole\% mixture of benzene in argon and oxygen, at an equivalence ratio of 0.190 . Total pressure in the microjet reactor was held constant throughout the runs at $344 \pm 2$ torr resulting in mean reactor residence times of $49 \pm 3 \mathrm{~ms}$.

\section{Data Analysis}

In this study mass spectral intensities from both the VUV-MS and a residual gas analyzer (RGA) are used to calibrate concentration measurements. To use the two sets of data for quantitative purposes the following issues must be taken into consideration : first, at the same concentration, a given species will produce different signal levels in the residual gas analyzer (RGA) spectra than any other species due to their differences in electron ionization cross sections and instrumental ion detection efficiencies. In the TOF spectra, a similar situation is encountered due to differences in photoionization cross sections at $10.5 \mathrm{ev}$, ion collection efficiencies, and detector sensitivities to ions of different velocities and chemical characteristics. In addition to this, the decrease in the total gas throughput to the analysis chamber, caused by decreases in gas density and discharge coefficient through the nozzle as the temperature is increased at constant pressure, must also be taken into account. Finally, a quantitative relationship between RGA and TOF data must be established.

\section{RGA Analysis}

In the RGA spectra the relationship between the partial pressure of a particular component in the mixture and the signal intensity at a given mass is

$$
P_{i}=\left(I_{i, m} / S i, m\right) r_{\text {nozzlc }}(T)
$$

where $P_{i}$ is the partial pressure of species $i$ at the reactor, $I_{i}$ is the RGA intensity (current in amps) measured at a selected mass used for quantifying species $i, S_{i, m}$ is an experimentally determined sensitivity factor for species $i$ at mass $m$ (amps per torr), and $f_{\text {nozzlc }}$ is a temperature dependent factor which takes into account the variations in nozzle throughput at different temperatures. 
The sensitivity factors $\left(S_{i, m)}\right.$ at room temperature are determined by measuring pressures at the microburner (MKS Baratron), rather than at the analysis chamber. The mass selected for analysis of a particular species is chosen such that there is unambiguous assignment (no overlap exists) with respect to any other fragments of parent species in the mixture. For the low concentration benzene oxidation mixtures reported here the choice is easy: mass 78 for benzene, 32 for oxygen, 28 for $\mathrm{CO}$ and 44 for $\mathrm{CO}_{2}$. Parent or frament ion overlaps from all possible hydrocarbon products are negligible here since they would produced signal levels much below the noise level of the RGA detector used, $0.5 \times 10^{-12}$ amps. This assumption was confirmed in the TOF analysis of the mixtures. The main overall reactions accessible with the residual gas analyzer are:

$$
\begin{aligned}
& \mathrm{C}_{6} \mathrm{I}_{6}+9 / 2 \mathrm{O}_{2} \rightarrow 6 \mathrm{CO}+3 \mathrm{H}_{2} \mathrm{O} \\
& \mathrm{C}_{6} \mathrm{H}_{6}+15 / 2 \mathrm{O}_{2} \rightarrow 6 \mathrm{CO}_{2}+3 \mathrm{H}_{2} \mathrm{O}
\end{aligned}
$$

Dircct water measurements are complicated because of its large adsorption cocfficient on the stainless steel walls of the analysis chamber, which excessively slows down attaining a steady state for reliable residual gas analysis. We obtain water concentrations from a mass balance of reactions (a) and (b).

At the low fuel concentrations in this studies (less than $4000 \mathrm{ppm}$ benzene in $\mathrm{Ar}$ ), the total number of moles can be considered constant within the overall uncertainty of our residual gas analysis (about 0.5 mole\%). The $\mathrm{f}_{\text {nozzle }}$ factor, therefore, is obtained with the assumption of constant Argon mole fraction from the RGA signal at mass 40:

$$
\mathrm{f}_{\mathrm{nIOzzl \textrm {lc }}(\mathrm{T})}=\mathrm{I}_{40} \mathrm{RGA}_{\left(25^{\circ} \mathrm{C}\right)} / \mathrm{I}_{40} \mathrm{RGA}_{(\mathrm{T})}
$$

The range of linear response from the RGA against pure gas inlet pressure was also determined during the early calibration procedure. This signal range spanned the range of up to $5 \times 10^{-10}$ amps. All experimental measurements were then made within this dynamic range.

$\mathrm{X}_{\mathrm{i}}$, the mole fraction of species $\mathrm{i}$ in the mixture, is then determined from the calculated total number of moles obtained from the accessible species in RGA analysis and the partial pressure information from Equation 1. 


\section{TOF Analysis}

Time-of-flight peak intensities are related to species concentrations in the reactor by the relation

$\mathrm{CTOF}_{(\mathrm{m}, \mathrm{l})}=\mathrm{f}_{\text {nozzlc }(\mathrm{T})}\left(\mathrm{CRGA}_{(\mathrm{bcnzcnc}, \mathrm{T})} / \mathrm{I}^{\mathrm{TOF}}(78, \mathrm{~T}, \mathrm{Wd}, \mathrm{g}) \mathrm{R}_{(\mathrm{m}, \mathrm{v}, \mathrm{Wa})} \mathrm{ITF}_{(\mathrm{m}, \mathrm{T} \mathrm{Wd} \mathrm{g})}\right.$

$\mathrm{C}^{\mathrm{TOF}}(\mathrm{m}, \mathrm{T})$ is the parts per million concentration of species with mass $\mathrm{m}$ at temperature $\mathrm{T}, \mathrm{f}_{\text {nozzle }}$ $(\mathrm{T})$ is the temperature factor defined in Equation (2), $\mathrm{CRA}^{\mathrm{RG}}$ (benzene, $\mathrm{T}$ ) is the benzene concentration (ppm) found with RGA analysis, $\mathrm{T}^{\mathrm{TOF}}(78, \mathrm{~T}, \mathrm{Wd}, \mathrm{g})$ and $\mathrm{ITOF}_{(\mathrm{m}, \mathrm{T}, \mathrm{Wd}, \mathrm{g})}$ are the observed integrated intensities of benzene and mass $m$ pea $\bar{k} s$, at laser radiant flux density $\mathrm{W}_{\mathrm{d}}$ (Watts $/ \mathrm{m}^{2}$ ) and detector gain $\mathrm{g}$, and $\mathrm{RTOF}_{\left(\mathrm{m}, \mathrm{V}_{\mathrm{io}}\right)}$ is an instrumental response factor given by

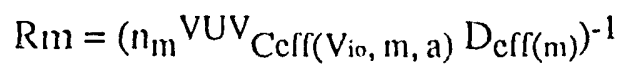

All the factors in this Equation (4) are normalized relative to benzene. $V_{U V}(m)$ is the ionization efficiency at $118 \mathrm{~nm}$ of species of mass $m, C_{e f f}(m, V i o, d)$ is the instrumental ion collection efficiency which corrects for any loses that may occur as the ions traverse the spectrometer to reach the detector of diameter $\mathrm{d}$ at a given ion optics voltage setting $\mathrm{V}_{\mathrm{io}}$, and $\mathrm{D}_{\mathrm{eff}}(\mathrm{m})$ is the micro channel plate detector secondary electron efficiency generation at mass $\mathrm{m}$.

Species values of $\operatorname{VUV}_{(\mathrm{m})}$ at $118 \mathrm{~mm}$ reported in the literature were used when available. We report estimated concentrations for larger than 78 amu species as if their $\mathrm{VUV}_{(\mathrm{m})}$ was identical to that of benzene. In most cases, specially when referring to aromatics, this assumption is only estimated to introduce an error less than $30 \%$ in the estimated concentrations. The theoreticallybased method we developed and use for estimating VUV photoionization efficiencies for any molecular species is discussed above.

In principle, ion trajectories for different masses introduced normal to the time-of-flight axis are not the same due to the une(gual kinetic energy component in this direction; even though molecular velocities in the thermalized reactor are distributed so that kinetic energies of species with different mass are the same, during the expansion this velocity distribution sharpens, and ions of different mass acquire different kinetic energies in the axial direction of the TOF tube. As a result, even when ions of a given mass might reach the detector at some set of conditions, this does not ensure that ions of all other masses will. Modeling of ion trajectories for masses between 10 and $600 \mathrm{amu}$ with the perpendicular sample introduction arrangement and the Einzel lens at the front end of the acceleration region of the TOF, resulted in all ions arriving at the 1 inch diameter microchannel 
plate detector when using appropriate ion optics settings and positioning of the ionization volume. Experimentally it is found that, after proper tuning of these parameters, a range of conditions is reached within which no variations of the ion intensities in a wide mass range (>400 amu) are observed. We assume that in this range the condition $\mathrm{C}_{\mathrm{eff}}(\mathrm{m}, \mathrm{Vio}, \mathrm{d})=1$ is reached for the mass range of interest.

As discussed in Section 4.2.1 above, $\mathrm{D}_{\mathrm{eff}(\mathrm{m})}$ also remains constant and equal to one for the mass range reported in this study, below 154 amu.

Finally we note that

$$
\mathrm{I}^{\mathrm{TOF}}(\mathrm{m}, \mathrm{T}, \mathrm{Wd}, \mathrm{g})=\mathrm{W}_{\mathrm{d}} g[\mathrm{C}]^{\mathrm{TOF}}(\mathrm{m}, \mathrm{T})
$$

where $[\mathrm{C}]^{\mathrm{TOF}}(\mathrm{m}, \mathrm{T})$ is the parts per million concentration of the neutral species in the laser ionization volume. From Equation (5), since all mass peaks detected (with the exception of acetone) correspond to one photon ionization, the I dependence on radiant flux density cancels out in Equation (3). The same is true for the gain $g$, given that the dynamic range of the microchannel plates used covers the range of experimental results. We also note that TOF peak intensities were experimentally found to be fairly independent of the exact location of the reactor expansion respect to the VUV focus volume, so that changes in the reactor positioning are not critical.

\section{Results and Discussion}

Figure 14 shows results for the residual gas analysis of $\mathrm{H}_{2} \mathrm{O}, \mathrm{CO}, \mathrm{CO}_{2}, \mathrm{O}_{2}$, benzene and $\mathrm{Ar}$ during oxidation of benzene in argon $\phi=0.19$ at temperatures from 885-995 K. Note the concentration axis is on a logarithmic concentration scale. The data cover the range of 6.5 (885 $\mathrm{K})$ to $80.5 \%(1000 \mathrm{~K})$ benzene conversion as noted above the residence time was $49 \pm 3 \mathrm{~ms}$. At temperatures higher than about $970 \mathrm{~K}$ the product yield to $\mathrm{CO}_{2}$ increases faster than that for $\mathrm{CO}$.

The low mass section of the raw VUV photoionization data for the same reactor conditions are presented in Figures 15 for temperatures increasing from 885-995 $\mathrm{K}$. Note the good resolution of low mass radicals $\mathrm{CH}_{3}$ and $\mathrm{C}_{3} \mathrm{H}_{3}$ at the higher temperatures shown. We emphasize that this spectra were taken at $10 \mathrm{~mJ}$ of $355 \mathrm{~mm}$ laser energy per shot, which yields $10^{1} \mu \mathrm{J}$ (order of magnitude) of $118 \mathrm{~nm}$ power. This corresponds to under $2 \mathrm{KWatts}$ of VUV power for ionization. Thus, the spectra are free from multiphoton framentation effects. Spot checks of signal dependence on power level support this observation. Of course, significant exceptions are acetone and possibly other oxygenated intermediates for which extremely fast internal conversion and/or ladder 
switching processes lead to VUV/UV fragmentation. Figure 15 illustrates the advantages of the sampling and analysis techniques used here; both stable and radical species can be analyzed in under 5 minutes of testing time.

In Figure 16 the concentrations of species with known ionization cross sections at $118 \mathrm{~mm}$ (data from Person and coworkers and references therein) are graphed. An error of up to $35 \%$ could be made in mass 40 concentration in this case. As noted above, several isomers are probable at most detected masses. In some cases a predominant isomer is likely and predictable from kinetic and thermodynamic arguments. Aside from this lack of isomer verification, the shortage of experimentally determined VUV photoionization and absorption cross sections for other species is the main limitation of the method at the present moment. Theoretically based correlation schemes for estimating ionization efficiencies are discussed above in Section 3.2 of this report. Results for detected hydrociabons from masses 50-154 amu are given on Figures 17 and 18. Concentrations are estimated for each mass assuming the estimated detection efficiency for the most stable species at the conditions of this study. The procedure followed to convert these intensities into species concentrations is outlined above. At the present time we assign a given mass to a single isotopic species, a choice which is made here on the basis of thermodynamics and kinetics arguments. Figure 19 shows estimated concentrations for the oxygenated hydrocarbons.

The TOF results show a decrease in the concentration of most hydrocarbon species above $970 \mathrm{~K}$, which is the same region where a higher relative increase of $\mathrm{CO}_{2}$ production when compared to $\mathrm{CO}$ is observed. Exceptions to this trend are some high (cresol, biphenyl) as well as low (butadiene, butadienyl radical and acetone) mass range species. This is consistent with these species being formed from phenyl radicals which reach higher concentrations as combustion intensifies due to the increased presence of both $\mathrm{H}$ and oxidizing radicals. Radical species are easily detected in our experiments, and as shown in Figure 20. The predominant hydrocarbon radicals detected were $\mathrm{C}_{6} \mathrm{H}_{7}$, cyclopentadienyl and 1,3 butadienyl radicals. The emergence of diacetylene signal in the high temperature run is consistent with different high temperature reaction channels that are accessible in the $1000 \mathrm{~K}$ range. $\mathrm{C}_{6} \mathrm{H}_{7}$ decomposition to acetylene and butadienyl radicals is apparent.

It is interesting to examine our results in the light of work by Glassman and coworkers (Venkat et al.). These investigators have used an adiabatic turbulent flow reactor with probe sampling and GC and GC/MS analysis to study the oxidation of lean $(\varnothing=0.39)$ benzene mixtures diluted in nitrogen, at temperatures between 1125 and $1185 \mathrm{~K}$. In their work the sampling was done along the axis of the reactor, corresponding to residence times between 18.5 and $92.5 \mathrm{msec}$. In our 
experiments the residence times, obtained from volumetric flow rates and the known reactor volume, were $49 \pm 3 \mathrm{msec}$. Before Glassman's work, most previous experimental studies of benzene oxidation had been carried out at much lower temperatures $(300-600 \mathrm{~K})$ with significantly longer reaction times. At these lower temperatures, the data suggests a mechanism in which formation of peroxide adduct initiates the oxidation process (Barnard and Ibberson).

In their paper, Glassman and coworkers suggested a high temperature mechanism in which phenyl radical oxidation to phenoxy radical, followed by $\mathrm{CO}$ ejection and cyclopentadienyl radical formation, are important reaction steps in the high temperature oxidation of benzene and its alkyl derivatives:

$\mathrm{C}_{6} \mathrm{H}_{5}+\mathrm{O}_{2} \rightarrow \mathrm{C}_{6} \mathrm{H}_{5} \mathrm{O}+\mathrm{O}$

$\mathrm{C}_{6} \mathrm{H}_{5} \mathrm{O}$ (Phenoxy) $\quad \rightarrow \mathrm{C}_{6} \mathrm{H}_{5}=\mathrm{O}$ (Ketocyclohexadienyl) (d)

$\mathrm{C}_{6} \mathrm{H}_{5} \mathrm{O}$ (Phenoxy) $\rightarrow \mathrm{C}_{6} \mathrm{H}_{5}$-OII (Phenol) (e)

$\mathrm{C}_{6} \mathrm{H}_{5}=\mathrm{O}-\mathrm{CO}+\mathrm{C}_{5} \mathrm{H}_{5}$ (Cyclopentadienyl)

In benzene oxidation, phenyl radicals are generated by reaction with molecular oxygen or $\mathrm{OH}, \mathrm{H}$ or $\mathrm{O}$ radical species. The isomerization reaction (d) competes with phenol formation (e) which becomes more predominant at higher temperatures. Efficient $\mathrm{CO}$ ejection from the Ketocyclohexadienyl also produces $\mathrm{C}_{5} \mathrm{H}_{5}(\mathrm{f})$.

The reaction then continues in an analogous manner to the phenyl and phenoxy radical steps (c) through (f), but now starting with cyclopentadienyl radical:

$\mathrm{C}_{5} \mathrm{H}_{5}+\mathrm{O}_{2} \rightarrow \mathrm{C}_{5} \mathrm{H}_{5} \mathrm{O}+\mathrm{O} \quad(\mathrm{g})$

$\mathrm{C}_{5} \mathrm{H}_{5} \mathrm{O}$ (Cyclopentadienoxy) $->\mathrm{C}_{5} \mathrm{H}_{5}=\mathrm{O}$ (Ketocyclopentadienyl) (h)

$\mathrm{C}_{5} \mathrm{H}_{5}=\mathrm{O}->\mathrm{CO}+\mathrm{C}_{4} \mathrm{H}_{5}(1,3$ Butadienyl)

Our results are consistent with the benzene oxidation mechanism proposed by Glassman. The lower temperatures at which we achieve similar benzene conversions for the same residence time 
(for $49 \mathrm{msec}$ we observe $45 \%$ conversion at $964 \mathrm{~K}$ vs. $37 \%$ at $1138 \mathrm{~K}$ ) are consistent with the different nature of the diluent gas (argon rather than nitrogen).

In the GC/MS analy'sis of Glassman and coworkers, the radical species participation in the mechanism was inferred from the stable species: e.i. cyclopentadienyl from cyclopentadiene, 1,3 butadienyl from 1,3 butadiene, etc. We present here direct evidence of the presence of these radicals in the high temperature oxidation of benzene. As in Glassman's work, however, we are unable to detect phenoxy or phenyl radicals. Reaction (c), the oxidation of phenyl radicals by molecular oxygen, at $1000 \mathrm{~K}$ is estimated to be fast, $E_{\mathrm{a}}=11 \mathrm{kcal}$ with a lower limit for preexponential factor $10^{9.2} \mathrm{l} / \mathrm{mole}$-sec. This would explain our failure to cietect phenyl radicals as the expected steady state concentration would be very low. Phenoxy/ketocyclohexadienyl (91 amu) was also not detected above 0.1 ppm concentration. This is likely because of fast decomposition to carbon monoxide and cyclopentadienyl. In addition, in this case, fast dissociative channels may be competing with direct photoionization. Phenol, however, is detected in appreciable quantities without the detection of possible photofragments. It is likely, therefore, that photofragmentation is not the reason for lack of detection of mass 91 but simply that the conversion to $\mathrm{C}_{5} \mathrm{H}_{5}$ is relatively fast. In Figure 20 it is interesting to note the changes in relative intensities of the cyclopentadienyl radical and cyclopentadiene. The radical is more prominent relative to the molecular species at the lower temperatures of this study.

The results in Figure 20 also present data showing production of both $\mathrm{C}_{3} \mathrm{H}_{3}$ and $\mathrm{CH}_{3}$ radicals. The $\mathrm{C}_{3} \mathrm{H}_{3}$ signal, however, is not quantitatively resolved at the lower temperatures due to interference from collisional ionization of the argon diluent at mass 40 .

The low signals for mass 81 and 82 are intriguing since the radical species $\mathrm{C}_{5} \mathrm{H}_{5} \mathrm{O}$ (2-cyclopentene-1-one, reaction (h)) and its stable molecular counterpart would appear at these masses. This is, however, the region of our TOF spectra where signals are strongly affected by impedance mismatch ringing between the collector plate of the detector and the digitizing scope. Therefore, we do not report concentrations for these species.

\section{Conclusion of Benzene Oxidation Study}

A microjet reactor coupled with a VUV-TOF mass spectrometer was used to study the moderate temperature (800-1000 K) oxidation of benzene. The high temperature oxidation mechanism proposed by Glassman was shown to predominate with cyclopentadienyl radical concentrations directly measured. Implications for coal pyrolysis/oxidation are that good species resolution and mechanistic insight to complicated systems can be easily obtained even in systems where both 
radical species, oxygenated and non-oxygenated hydrocarbons are important and their concentrations vary by many orders of magnitude.

\subsection{OVERALL TECIINICAL CONCLUSION AND RECOMMENDATIONS}

In this work we have demonstrated the utility of VUV photoionization coupled with time-of flight mass spectrometry as a powerful and characterizable technique for analysis of complex reacting hydrocarbon mixtures typical of those found in coal processing chemistry. A major advantage of this technique is that all stabile and labile masses can be viewed simultaneously and nondestructively at sub-ppm concentrations in real time. Issues relating to calibration of ion yields and detectability have been addressed and found to be considerably simplified when compared to other ionization/detection schemes. One limitation of the technique could be the dearth of information on photoionization yields for hydrocarbons in the VUV. While this can be addressed by using experimental calibrations, we have also developed a theoretically-based method for correlating VUV photoionization yields, similar to one recently proposed by Koizumi (1991), making use of simultaneously obtained photoabsorption and photoionization cross section of alkanes, alkenes, alcohols and ethers (over the range of $\mathrm{C} 1$ to $\mathrm{C} 6$ ). Thus, unlike with hot-wire EI ionization, where there is always a spread of electron energies, the ionization wavelength is monoenergetic and for many cases theoretically-based estimates of relative photoionization yields can be obtained directly. Tuning of the ionization wavelength can be used to obtain a degree of species selectivity, to minimize fragmentation probability, and to verify theoretically-based correlation estimates for photoionization efficiency.

Suggestions for future work include continued development of simple methods for obtaining compound selectivity. Although this is not obtained with a single wavelength, single mass filter instrument, simple methods are available limited only by the degrees of freedom in information gathered to characterize a complex reacting sample. Selectivity for structural studies can be provided using simultaneous pulsed EI (low conversion data only), tandem mass filters (as shown in Figure 7), variable wavelength VUV photoionization, or by a number of other spectroscopies in the sampled product beam. In addition, for a combustor/process reactor application a single wavelength, single mass filter instrument is inexpensive, robust, extremely sensitive and would provide valuable information for process monitoring and control. 
Table 1

RELATIVE CONCENTRATIONS OF SELECTED PRODUCT SPECIES DURING THE PYROLYSIS OF ALLENE 2ms, 600 Torr.

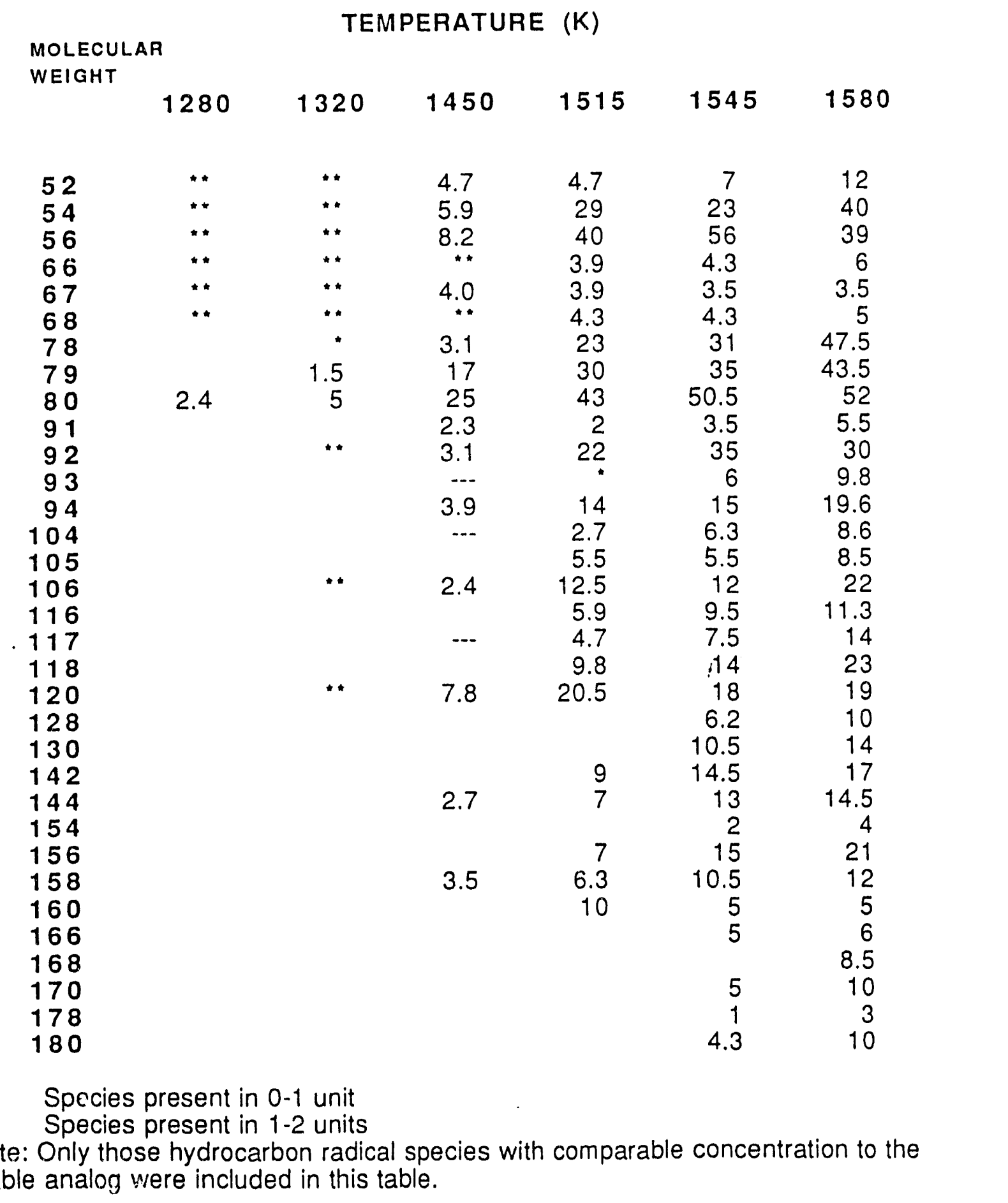


Table \#2:

\section{Theoretical Estimation of Rate Constant for Dimerization of Allene to 1,2 DMCB}

$$
2(\mathrm{C}=\mathrm{C}=\mathrm{C}) \cdots[]^{\ddagger}-->
$$

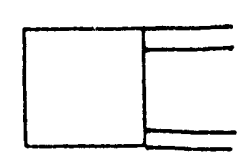

$\begin{array}{llll}\Delta H_{f} & 2 \times(45.9) & \sim 107 & 47.24\end{array}$ $(\mathrm{kcal} / \mathrm{mol})$

$\mathrm{S}_{300 \mathrm{~K}} \quad 116.6$

74.12 (cal/mol.K)
$A^{*} \sim 1.0 \times 10^{7} \mathrm{l} / \mathrm{mol} \mathrm{sec} @ 298 \mathrm{~K}$ (estimate from Gajewski and Shih) $E^{\ddagger} \sim 15 \mathrm{kcal} / \mathrm{mol}$

\section{QRRK estimate \\ Argon bath gas, 1 atm}

$\begin{aligned} k_{\text {dimerization }} & \approx 4.3 \times 10^{2} \mathrm{~cm}^{3} / \mathrm{mol} \mathrm{sec} @ 700 \mathrm{~K} \\ & \approx 1.9 \times 10^{3} \mathrm{~cm}^{3} / \mathrm{mol} \mathrm{sec} @ 800 \mathrm{~K}\end{aligned}$ 
Table 3: Possible Combinations of PAH + PAH to form High Mass Dimers $+\mathrm{H}_{2}$

\begin{tabular}{|c|c|c|c|c|c|c|c|c|c|c|c|c|c|}
\hline $\begin{array}{l}\text { Dimer: } \\
\text { Monomer }\end{array}$ & 408 & 420 & 438 & 468 & 482 & 527 & 526 & 528 & 542 & 544 & 586 & 600 & 602 \\
\hline 78 & 332 & 344 & 362 & 392 & 406 & & & & & & & & \\
\hline 92 & 318 & 330 & 348 & 378 & 392 & & & & & & & & \\
\hline 104 & 306 & 318 & 336 & 366 & 380 & & & & & & & & \\
\hline 106 & 304 & 316 & 334 & 364 & 478 & & & & & & & & \\
\hline 116 & & 306 & 324 & & & & & & & & & & \\
\hline 120 & & 302 & 320 & 350 & 364 & & 408 & & & & & & \\
\hline 128 & & & 312 & & & & 400 & 402 & & & & & \\
\hline 135 & & & & & & 394 & & & & & & & \\
\hline 142 & 268 & & & 328 & & & & & & & & & \\
\hline $\begin{array}{l}152 \\
154\end{array}$ & & & & 318 & 332 & & & & & & & & \\
\hline $\begin{array}{l}154 \\
166\end{array}$ & 256 & 268 & & 316 & 330 & & 374 & 376 & 390 & 392 & & & \\
\hline $\begin{array}{l}166 \\
168\end{array}$ & & 256 & & 304 & 318 & & 362 & 364 & 378 & 380 & & & \\
\hline $\begin{array}{l}168 \\
178\end{array}$ & 242 & 254 & & 302 & 316 & & 360 & 362 & 376 & 378 & & & \\
\hline 178 & & & & 292 & 306 & & 350 & 352 & 366 & & & & \\
\hline 180 & 230 & 242 & & & 304 & & 348 & 350 & 364 & 366 & 408 & & \\
\hline 190 & & & & & & & 338 & 340 & & & & & \\
\hline 192 & 218 & 230 & & 278 & 292 & & 336 & 338 & $\begin{array}{l}352 \\
350\end{array}$ & 352 & 394 & 408 & \\
\hline 194 & 216 & 228 & & 276 & & & 334 & 336 & $\begin{array}{r}350 \\
344\end{array}$ & 386 & 400 & 402 & \\
\hline 202 & & & & 268 & & & 326 & 328 & $\begin{array}{l}344 \\
340\end{array}$ & & 384 & & 400 \\
\hline 204 & 200 & 218 & & 266 & & & 324 & 326 & $\begin{array}{l}340 \\
338\end{array}$ & 340 & & 396 & \\
\hline 206 & & 216 & & & 278 & & 322 & 324 & $\begin{array}{l}338 \\
328\end{array}$ & 330 & 372 & 386 & 388 \\
\hline 216 & & $\begin{array}{l}206 \\
204\end{array}$ & & 254 & 268 & & 312 & & 326 & 328 & & 384 & 386 \\
\hline 218 & & 204 & & 252 & 266 & & 310 & $\begin{array}{r}312 \\
304\end{array}$ & 318 & 320 & 362 & 376 & 378 \\
\hline 226 & & & & & & & 302 & $\begin{array}{l}304 \\
30 ?\end{array}$ & 316 & 318 & 360 & 374 & 378 \\
\hline 228 & & 194 & & 242 & 256 & & & 302 & & & & & 376 \\
\hline 230 & 180 & 192 & & 240 & 254 & & & & & 316 & 358 & 372 & 374 \\
\hline 240 & & & & 230 & & & & & 304 & 306 & 348 & 362 & 364 \\
\hline 242 & 168 & 180 & & 228 & & & & & 302 & 304 & 346 & 360 & 362 \\
\hline 252 & & & & 218 & & & 276 & 278 & 292 & & 336 & 350 & 352 \\
\hline 254 & & 168 & & 216 & 230 & & & 276 & & 292 & 334 & 348 & 350 \\
\hline 256 & & 166 & & & 228 & & & & & & 332 & 346 & 348 \\
\hline 266 & & & & 204 & 218 & & & & 278 & & 322 & 336 & 338 \\
\hline 268 & 142 & 154 & & 202 & 216 & & & & 276 & 278 & 320 & 334 & 336 \\
\hline 276 & & & & 194 & & & 252 & 254 & 268 & & 312 & 326 & 328 \\
\hline 278 & & & & 192 & 206 & & & 252 & 266 & 268 & 310 & 324 & 326 \\
\hline
\end{tabular}




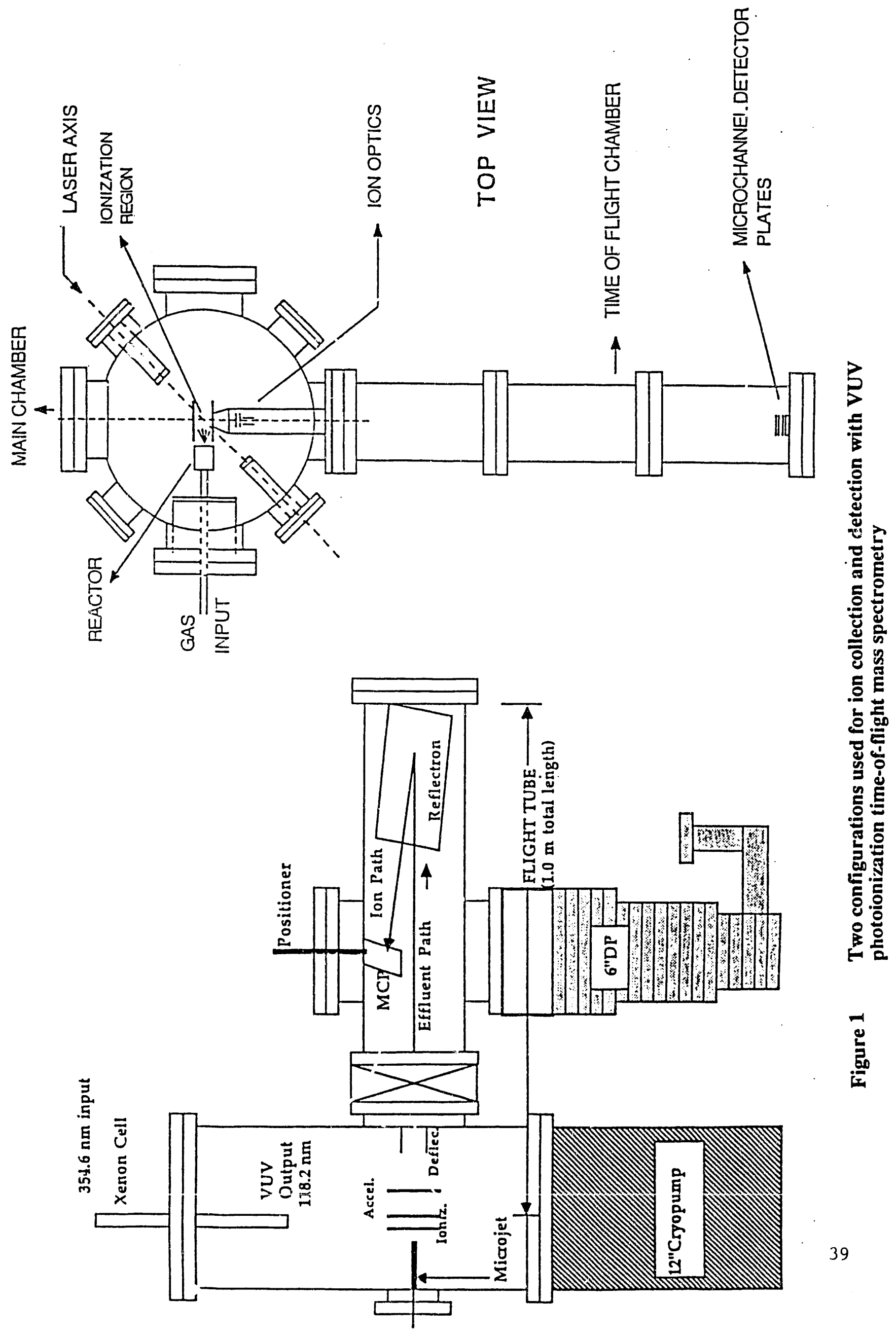




\section{Propane}

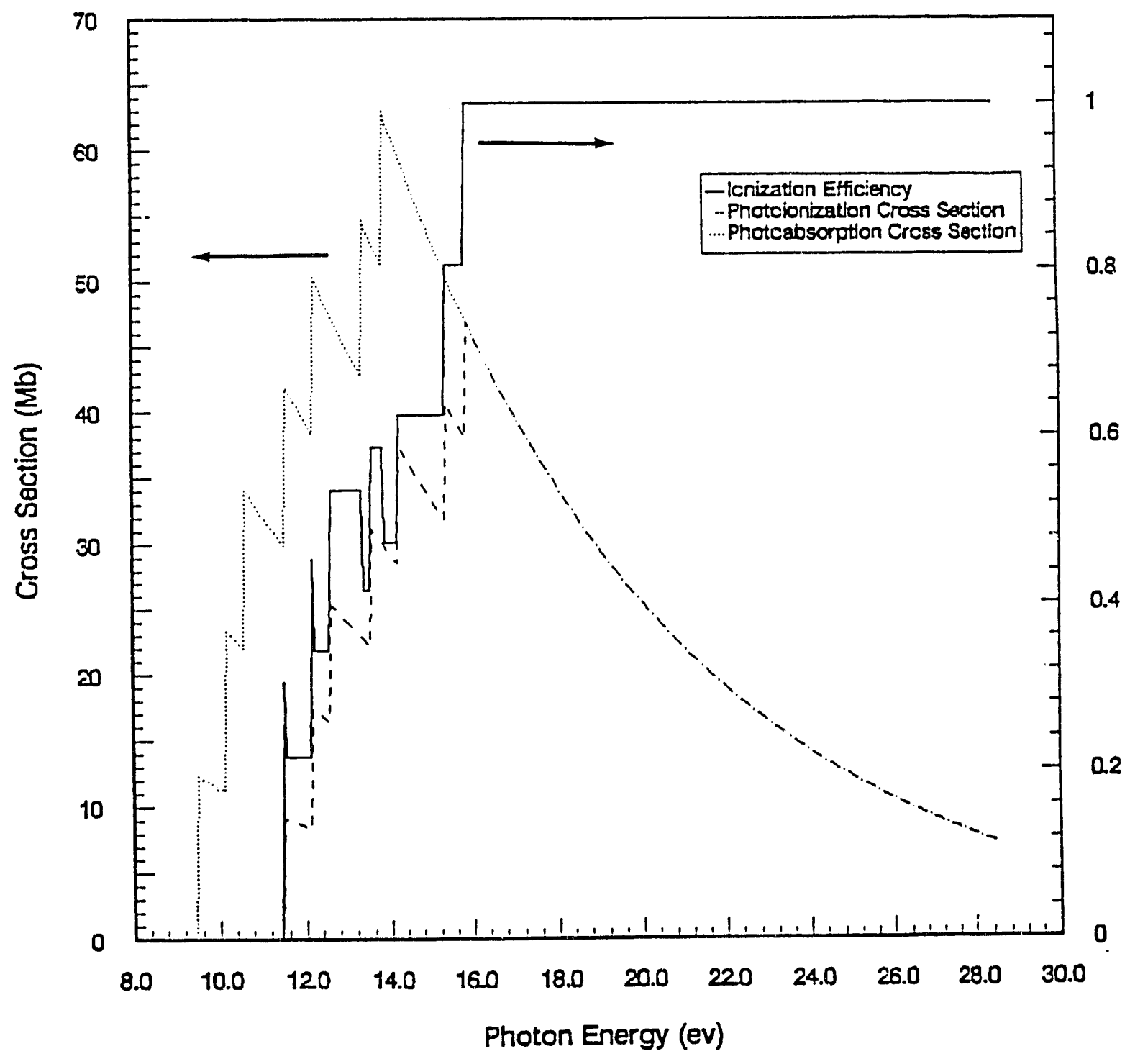

Figure 2 Estimated VUV photoionization efficiency as a function of ionizing photon energy for propane. 


\section{Propene}

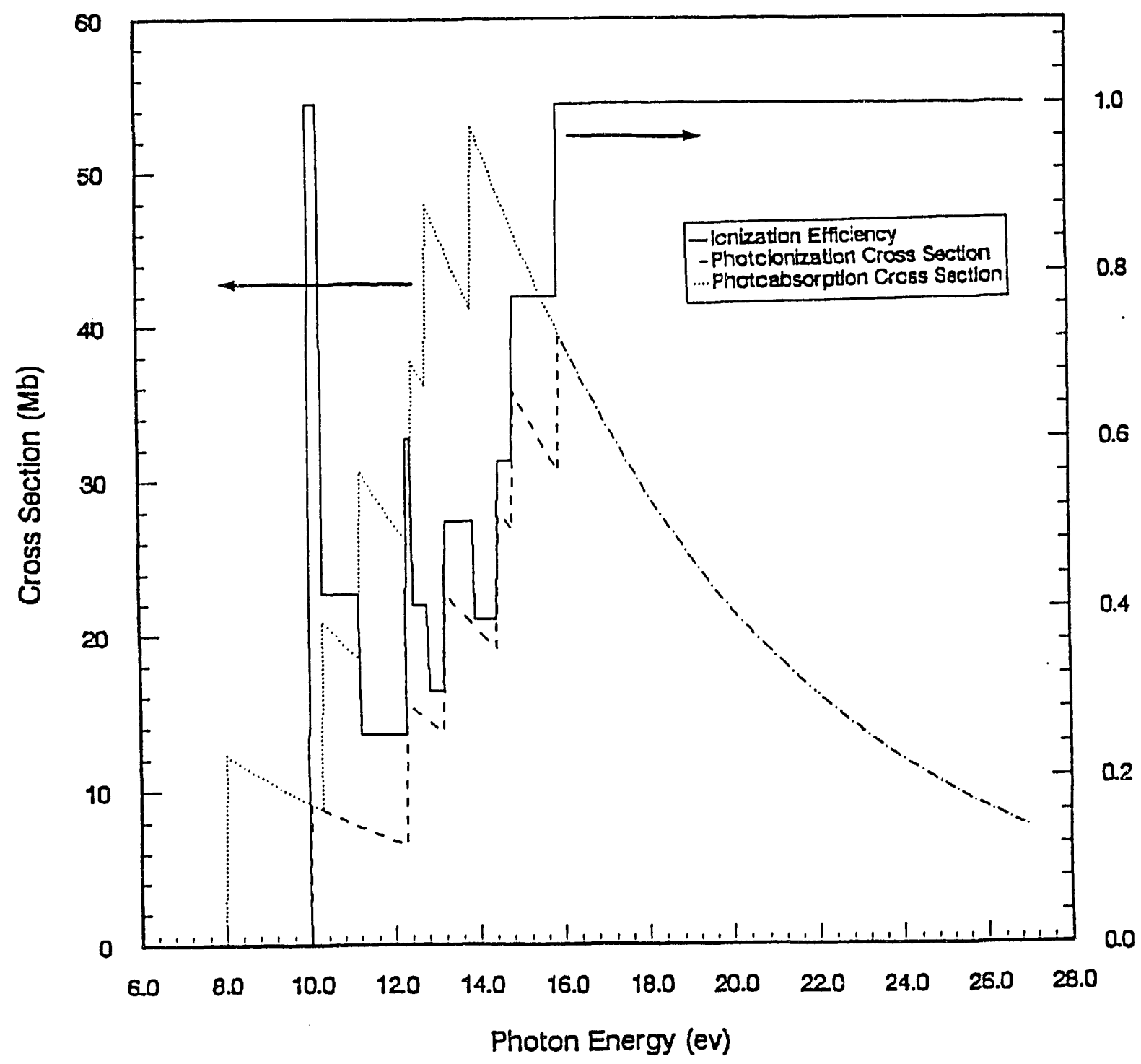

Figure 3 Estimated VUV photoionization efficiency as a function of ionizing photon energy for propene. 

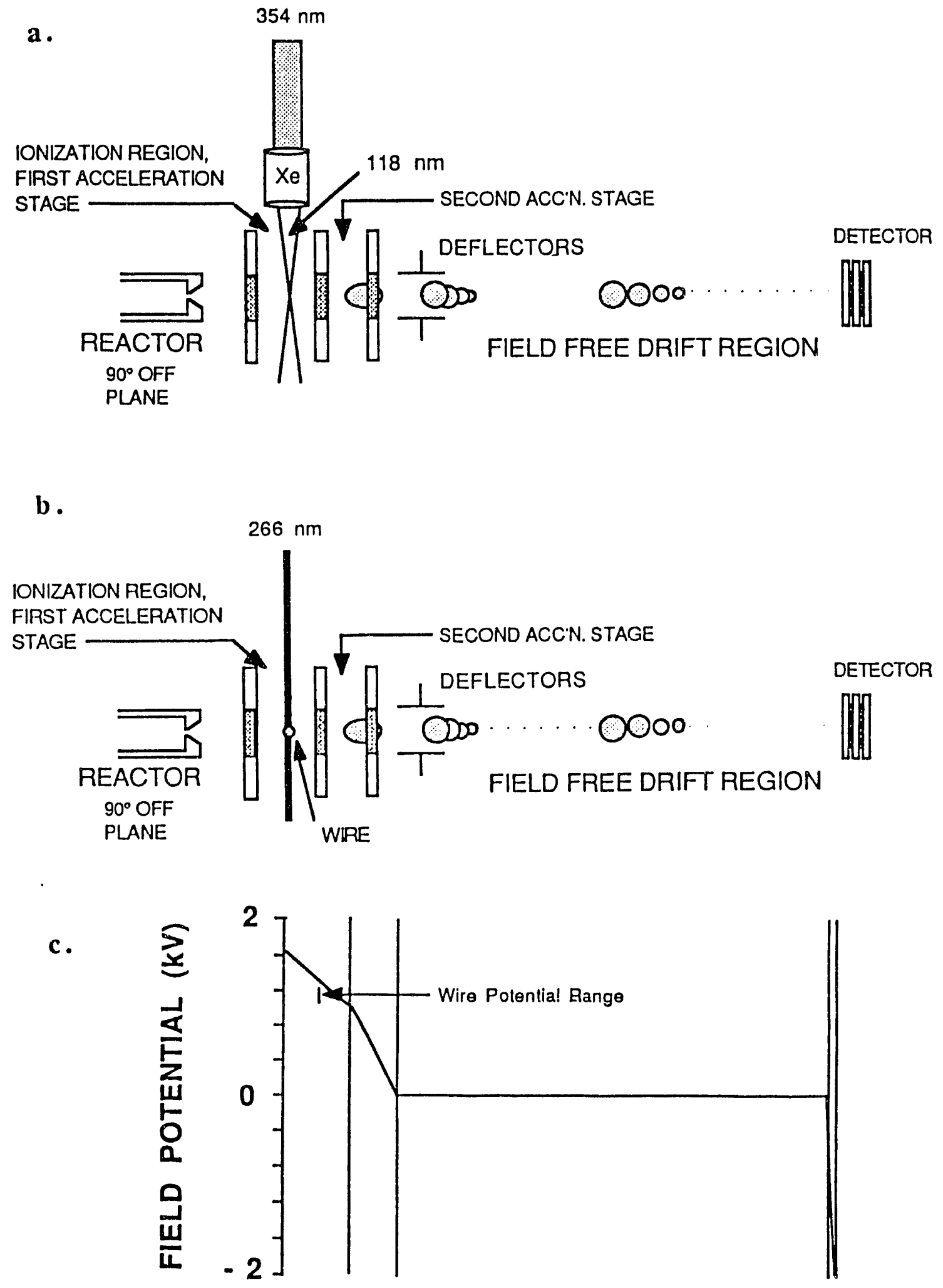

\section{ION POSITION}

Figure 4 Schematic of the VUV-photoionization / UV-LDEI TOF mass spectrometer. a) VUV-Photoionization mode. b) UV-LDEI mode. c) Potentiai energy profile of the acceleration and drift region. 


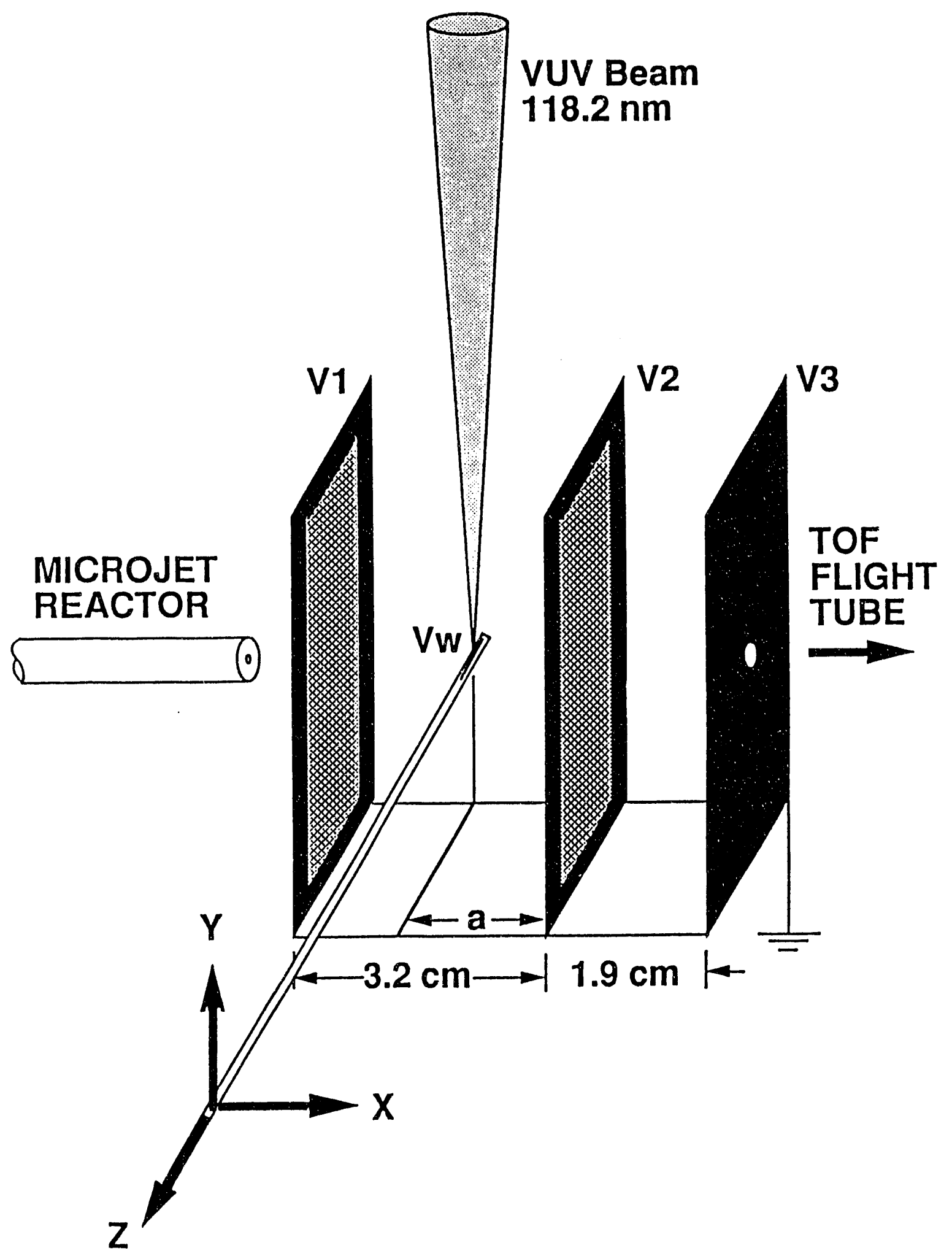

Figure 5 Schematic of the VUV-photoionization / VUV-LDEI TOF mass spectrometer. Typical operating condition: $V_{1}=+2000 \mathrm{~V}, \mathrm{~V}_{2}=$ $+1000 \mathrm{~V}, \mathrm{~V}_{3}=0 \mathrm{~V}, \mathrm{~V}_{\mathrm{W}}=+1400 \mathrm{~V}, \mathrm{a}=1.6 \mathrm{~cm}$. Wire translates in the $\mathrm{Z}$ direction. 


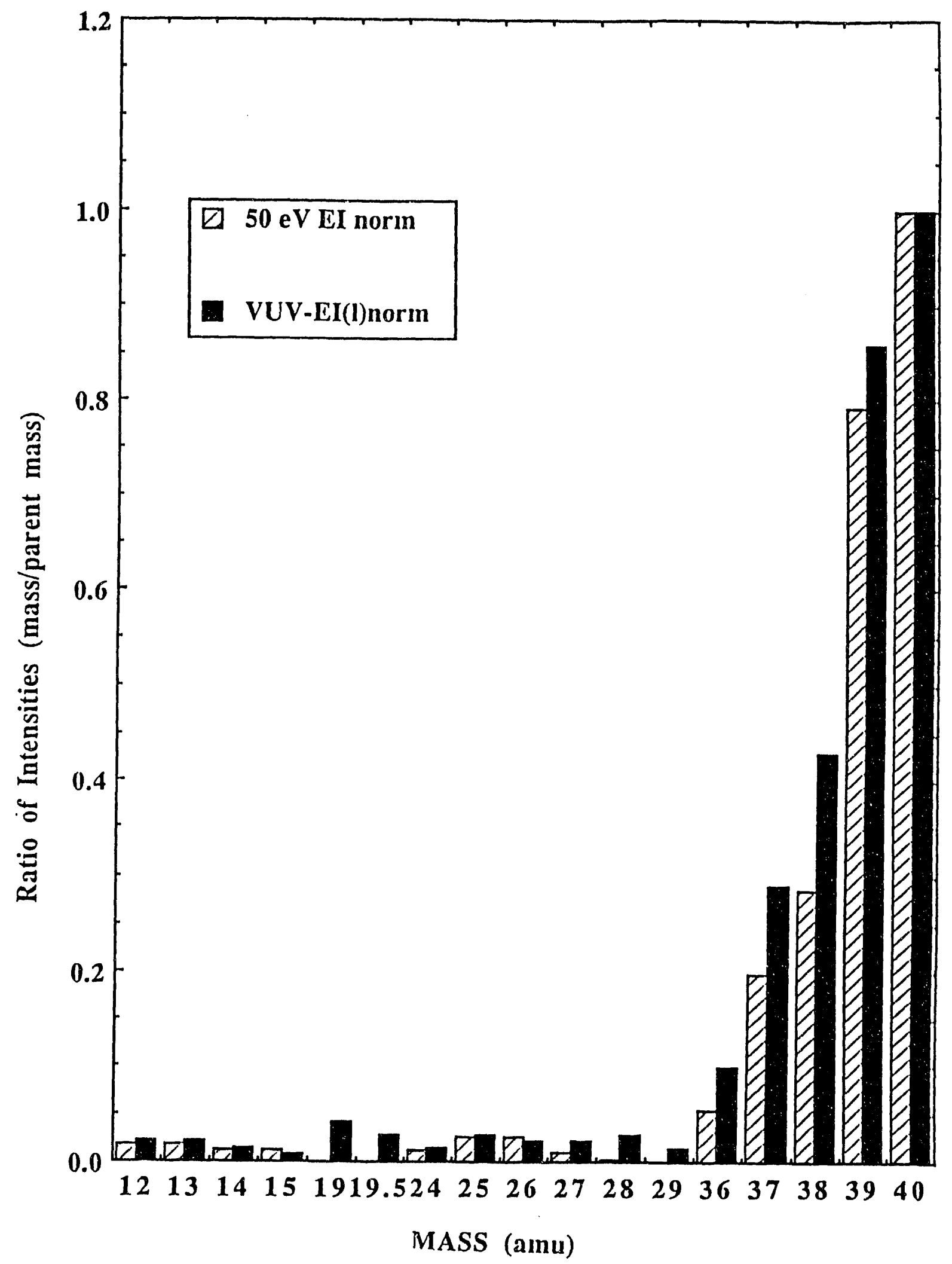

Figure 6 Mass spectral distribution of methyl acetylene, normalized by parent mass intensity, comparing VUV-LDEI and a standard $50 \mathrm{eV}$ electron ionization source. 


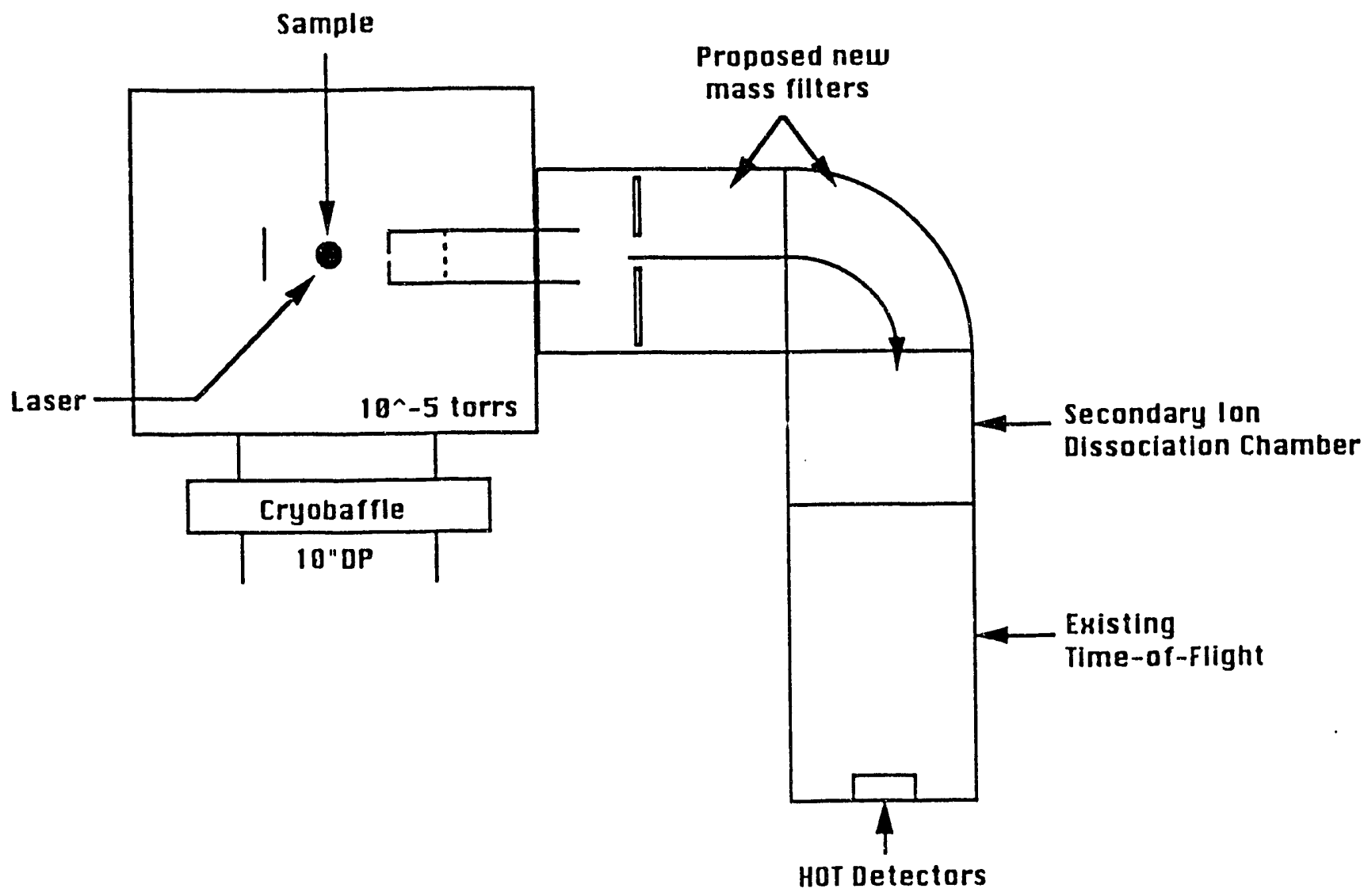

Figure 7 Schematic of proposed VUV-photoionization TOF Mass Spectrometer with additional mass filter for isomeric compound differentiation. 
Figure 8 Mass spectra taken during allene pyrolysis from microreactor at $2 \mathrm{~ms}$ residence time and temperalures from 1320 to $1660 \mathrm{~K}$ (mass 40 is off-scale for 1320 to $1400 \mathrm{~K}$ ).

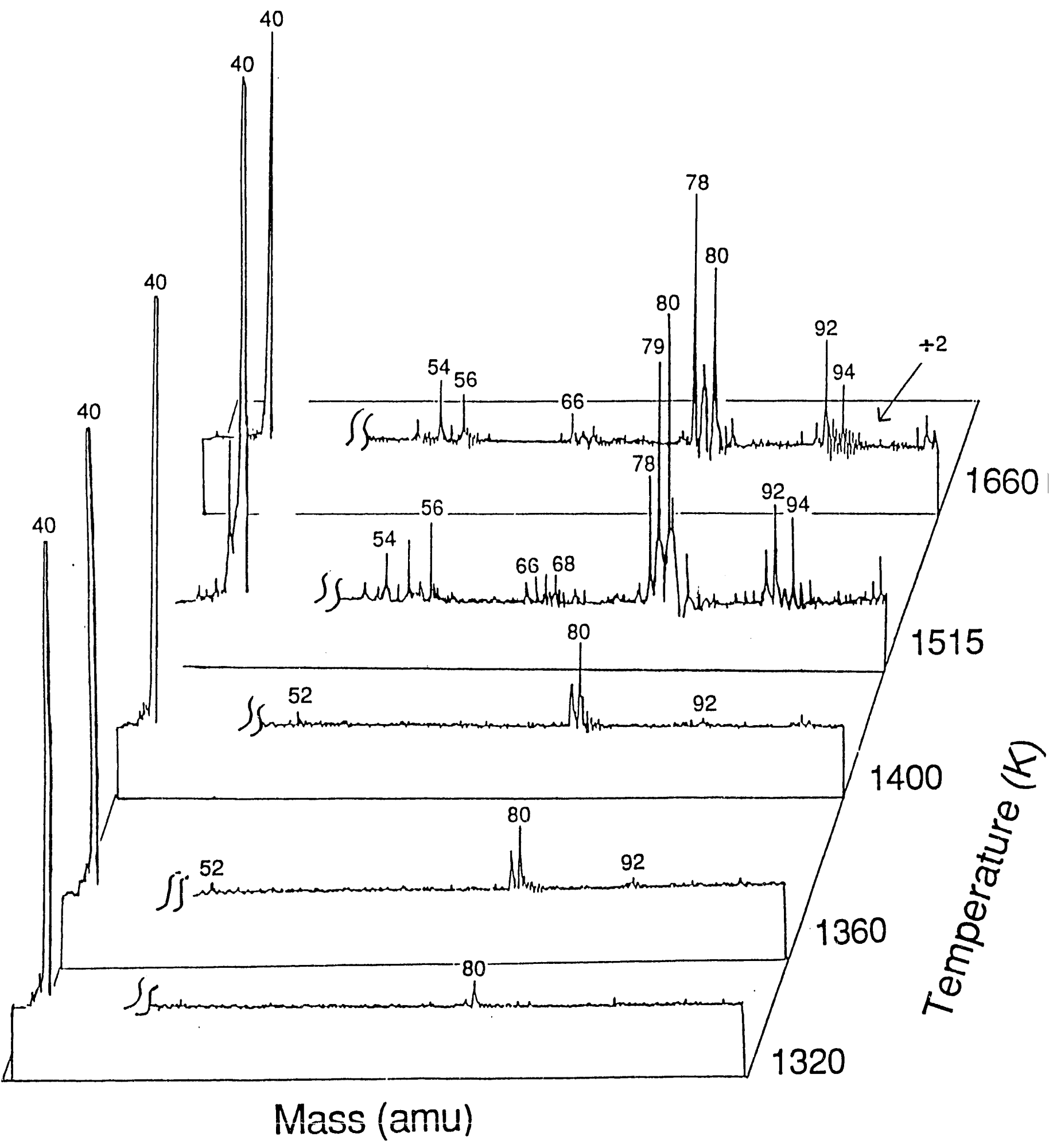




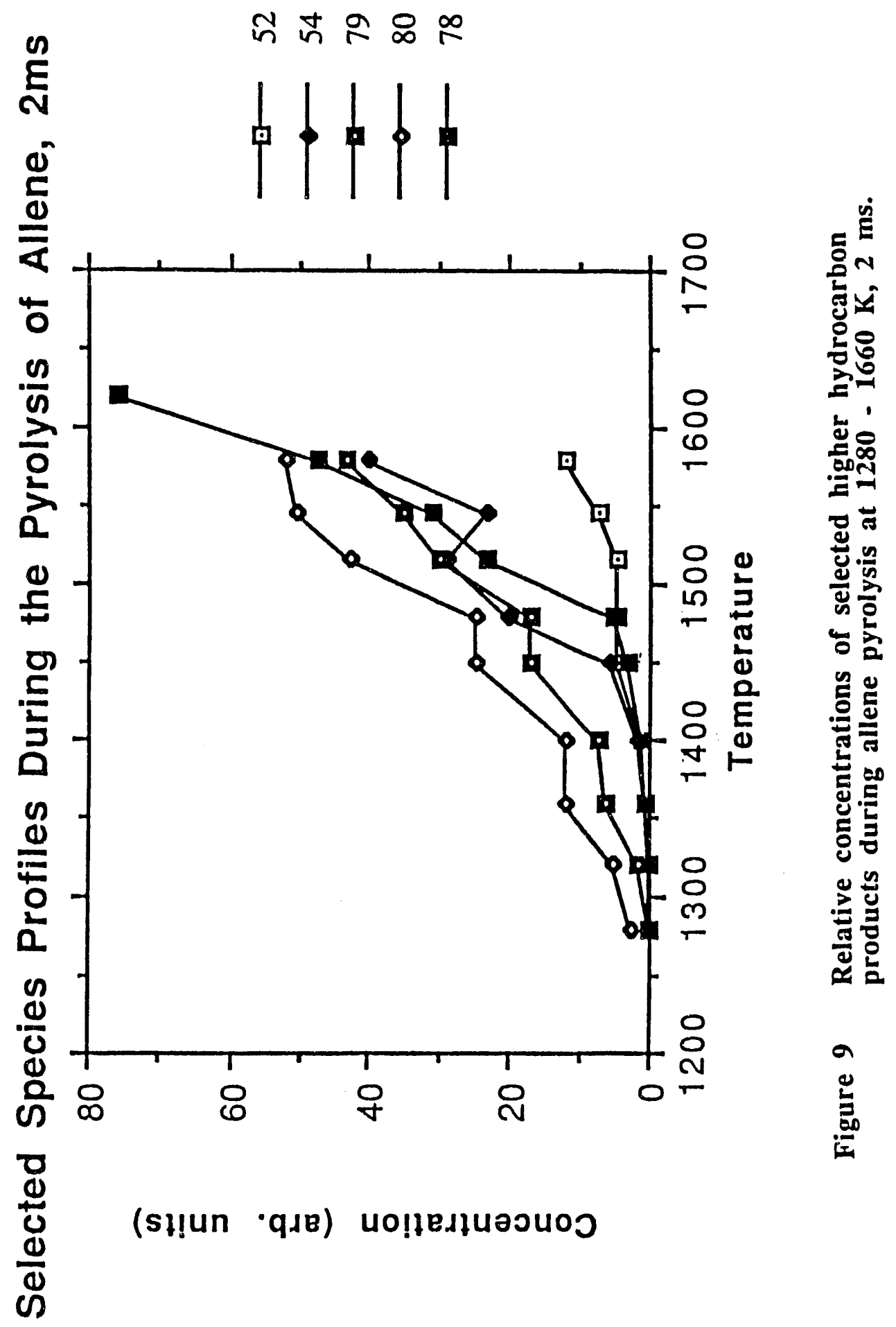


Figure 10 Overview spectra of product abundances during neat allene pyrolysis, $1495 \mathrm{~K}, \mathrm{P}^{\prime}=915$ Torr.

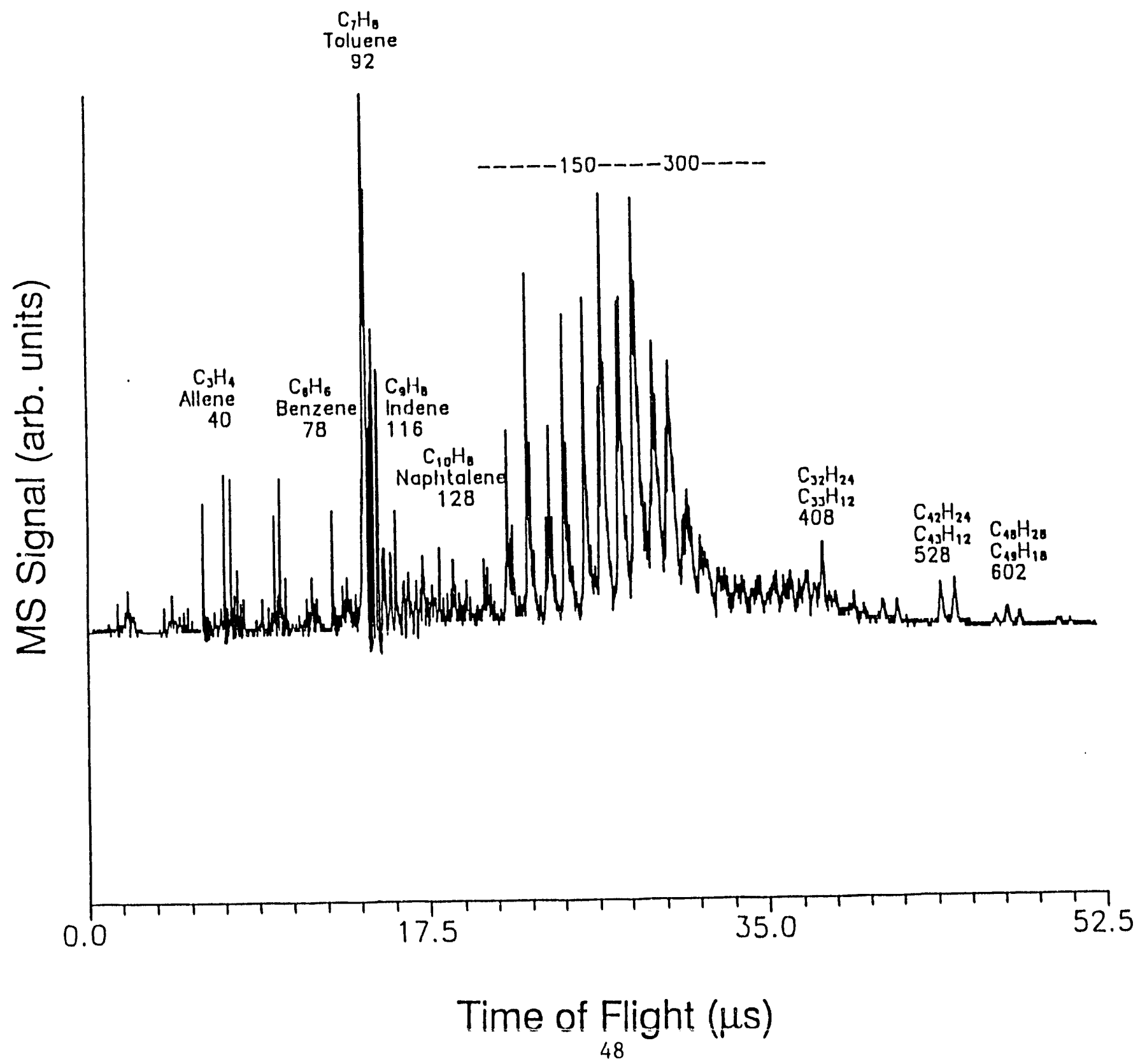


Figure 11 Detail spectra of product abundances in the mass range 152300 amu during neat allene pyrolysis, $1495 \mathrm{~K}, \mathrm{P}=915$ Torr.

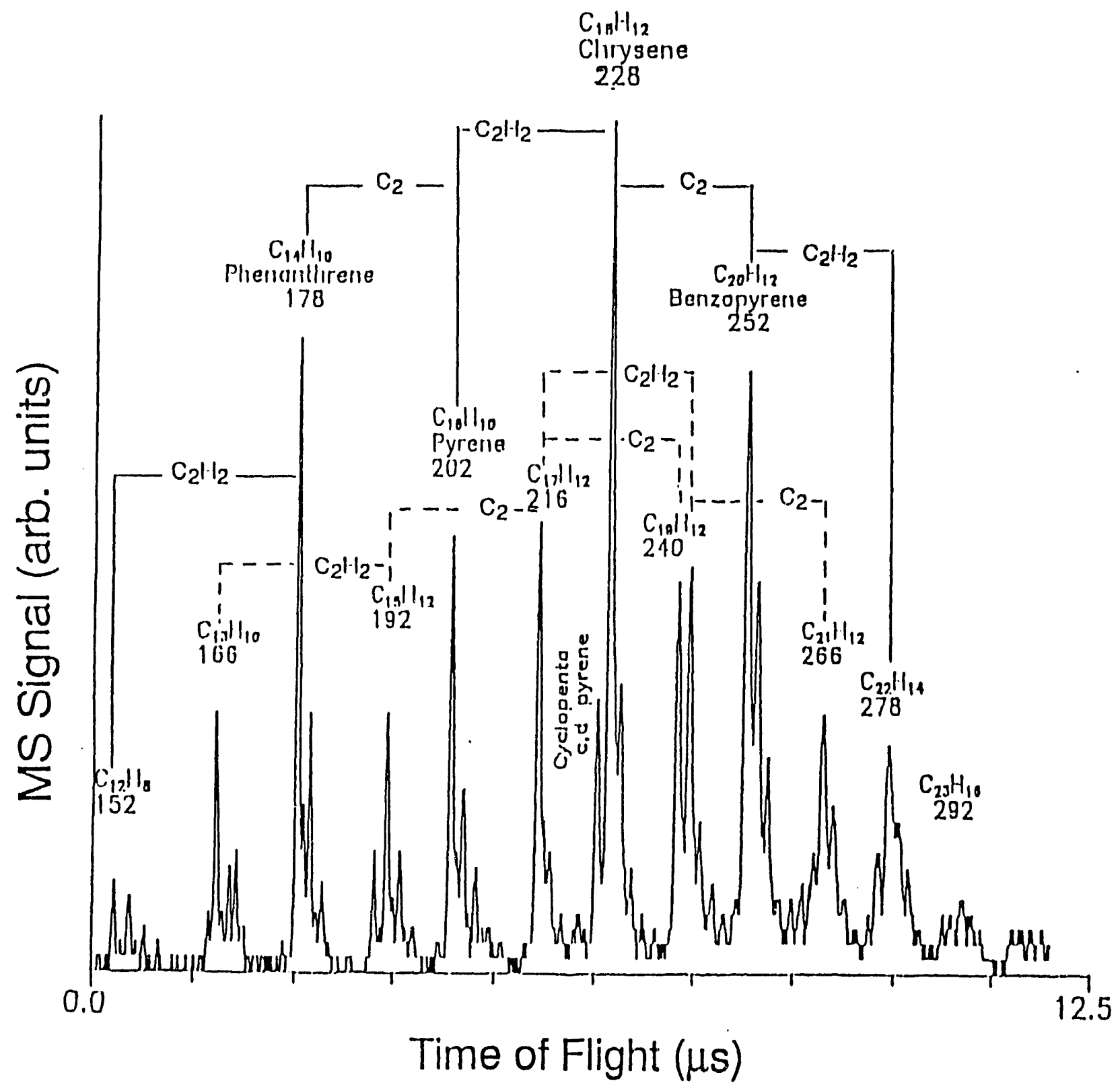


Figure 12 Detail spectra of product abundances in the mass range 300 408 amu during neat allene pyrolysis, $1495 \mathrm{~K}, P=915$ Torr.

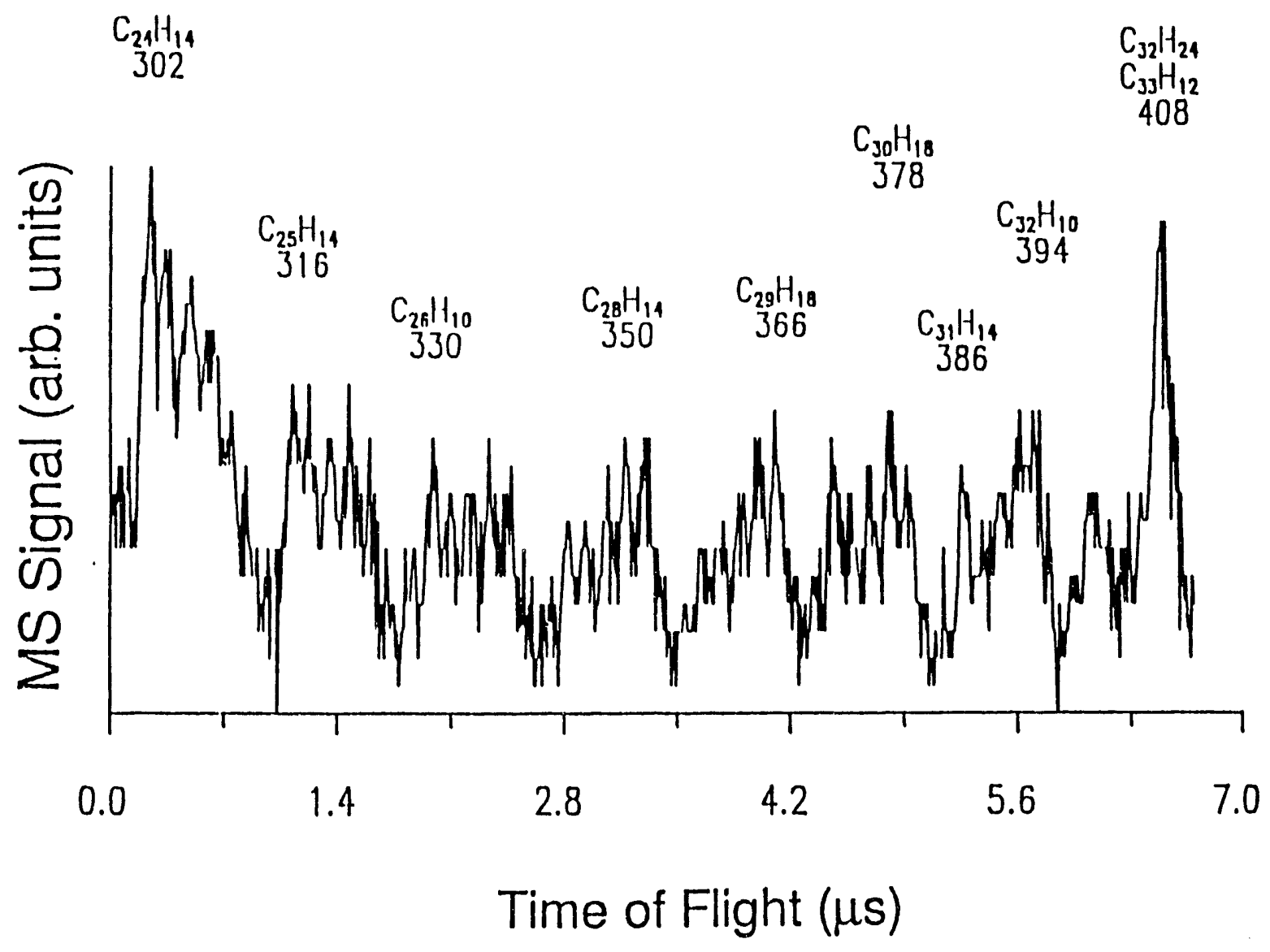


Figure 13 Detail spectra of product abundances in the mass range 408662 amu during neat allene pyrolysis, $1495 \mathrm{~K}, \mathrm{P}=915$ Torr.

$$
\begin{gathered}
\mathrm{C}_{32} \mathrm{H}_{24} \\
\mathrm{C}_{33} \mathrm{H}_{12} \\
408
\end{gathered}
$$

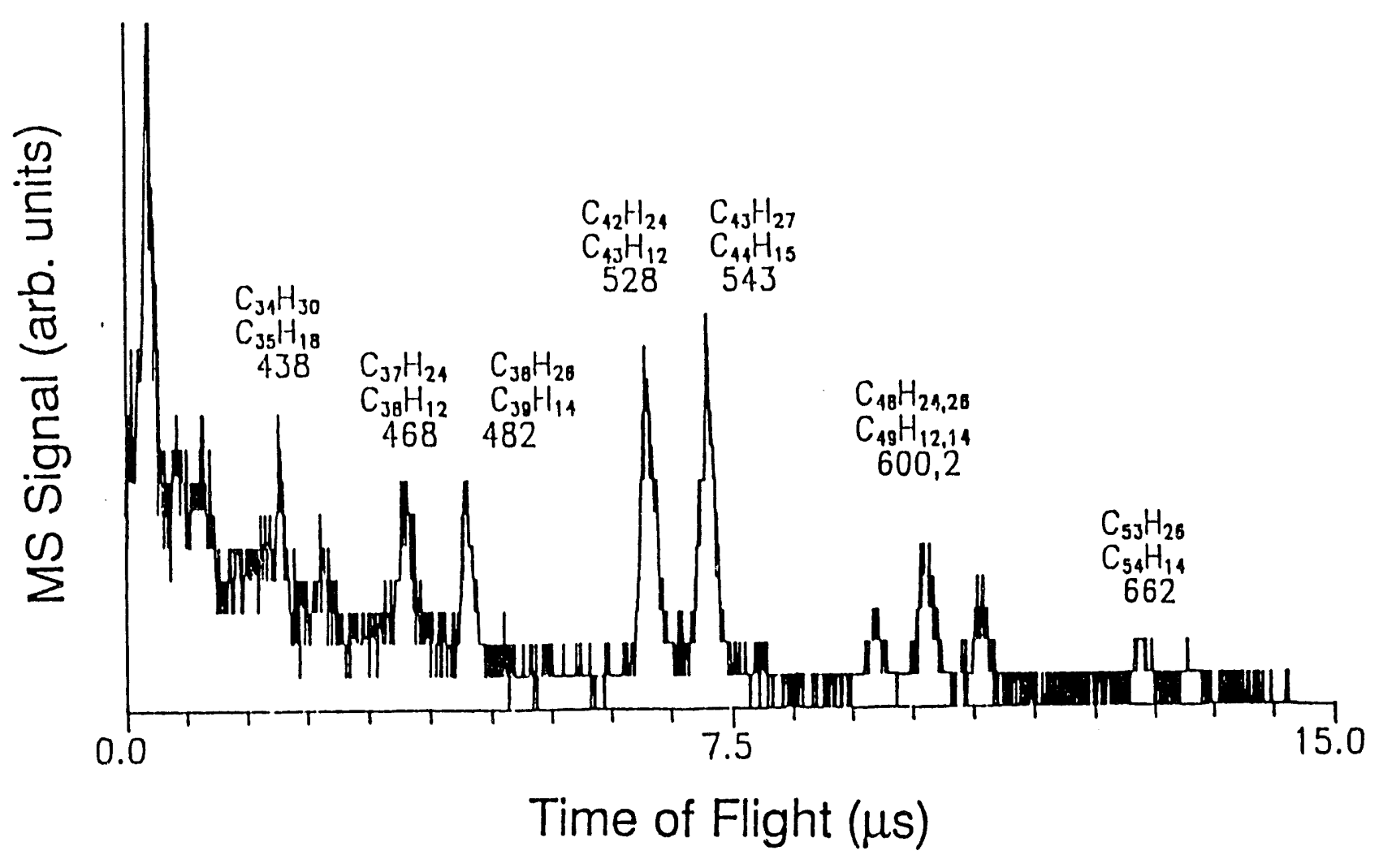




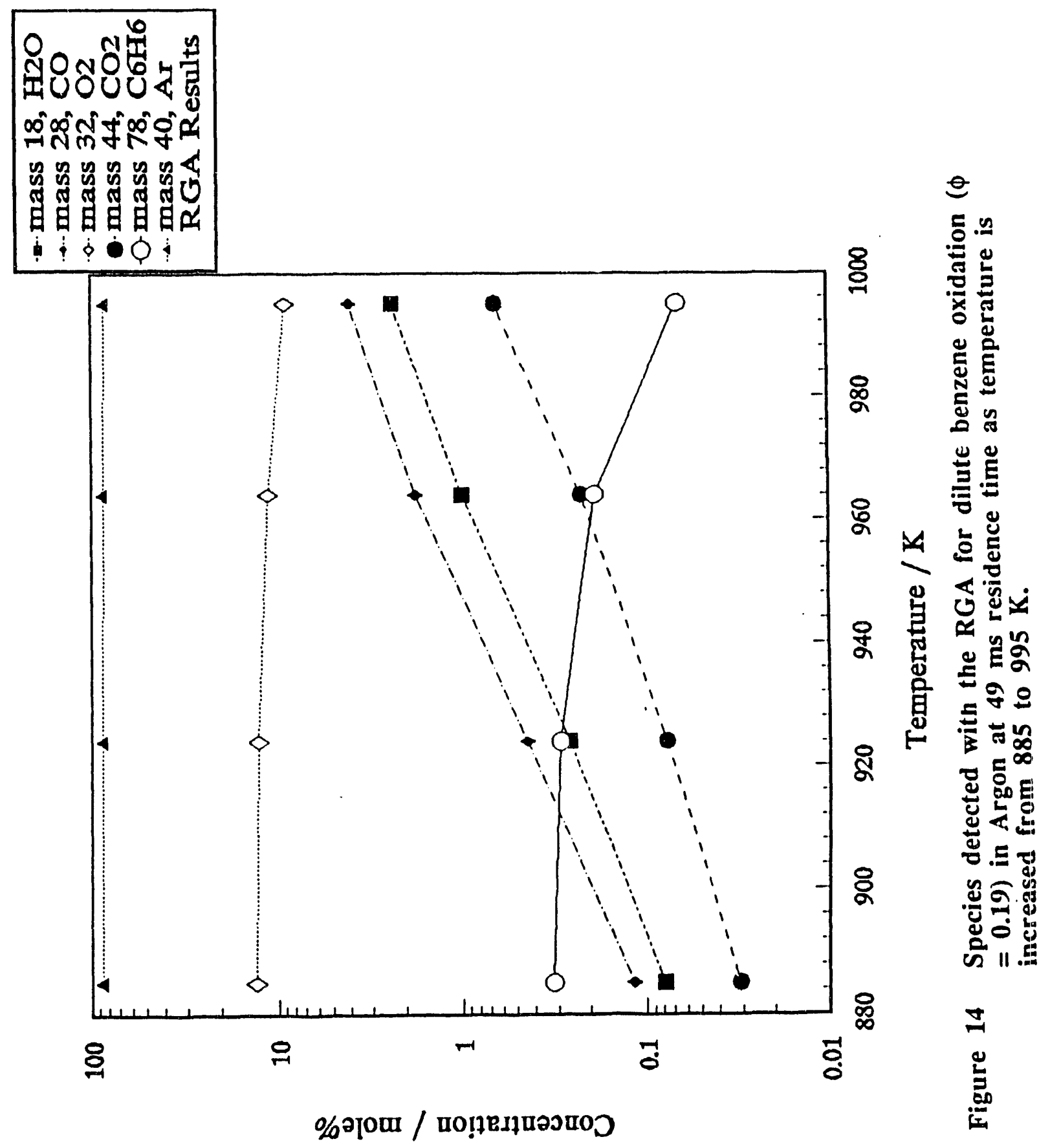




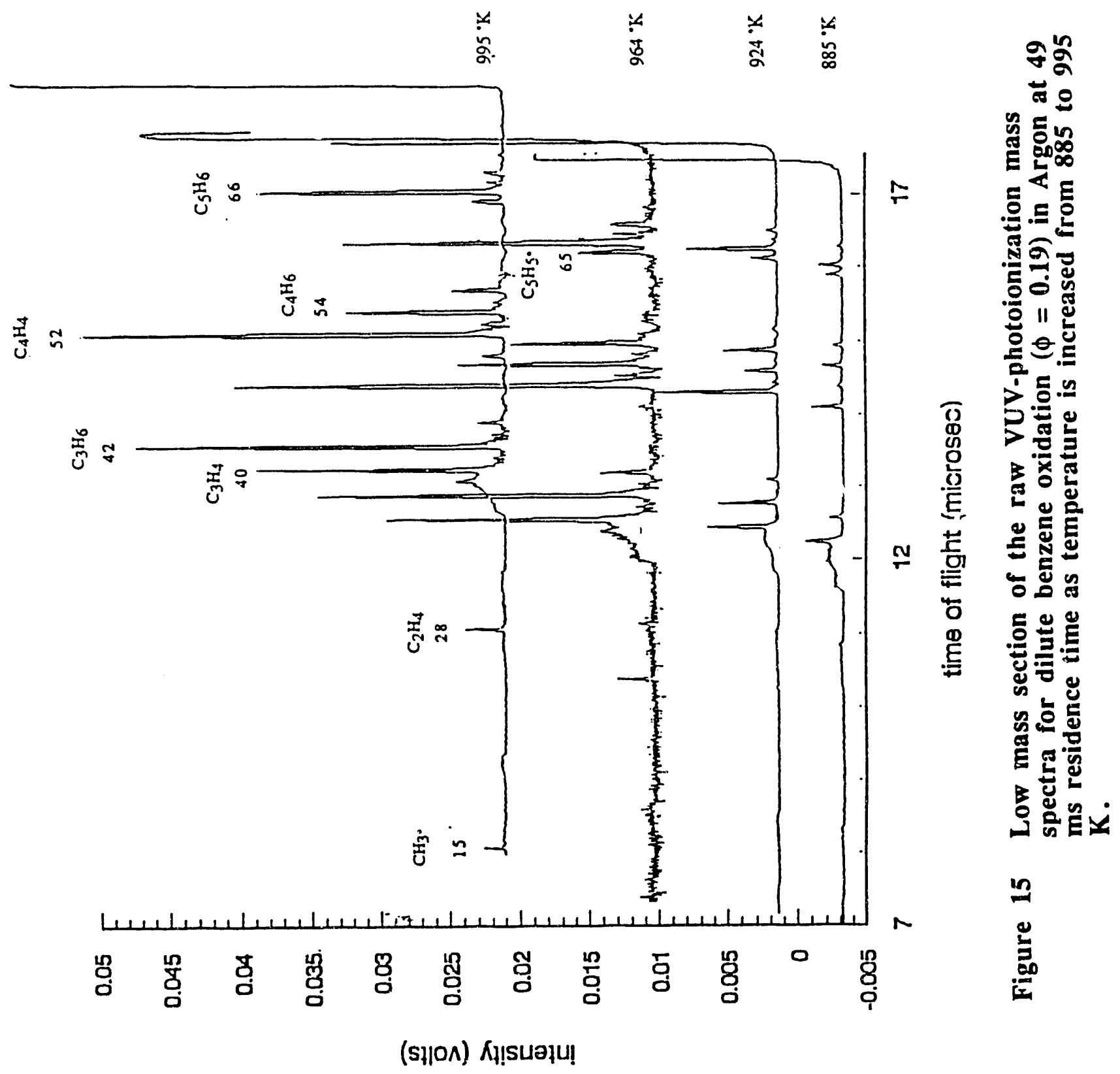




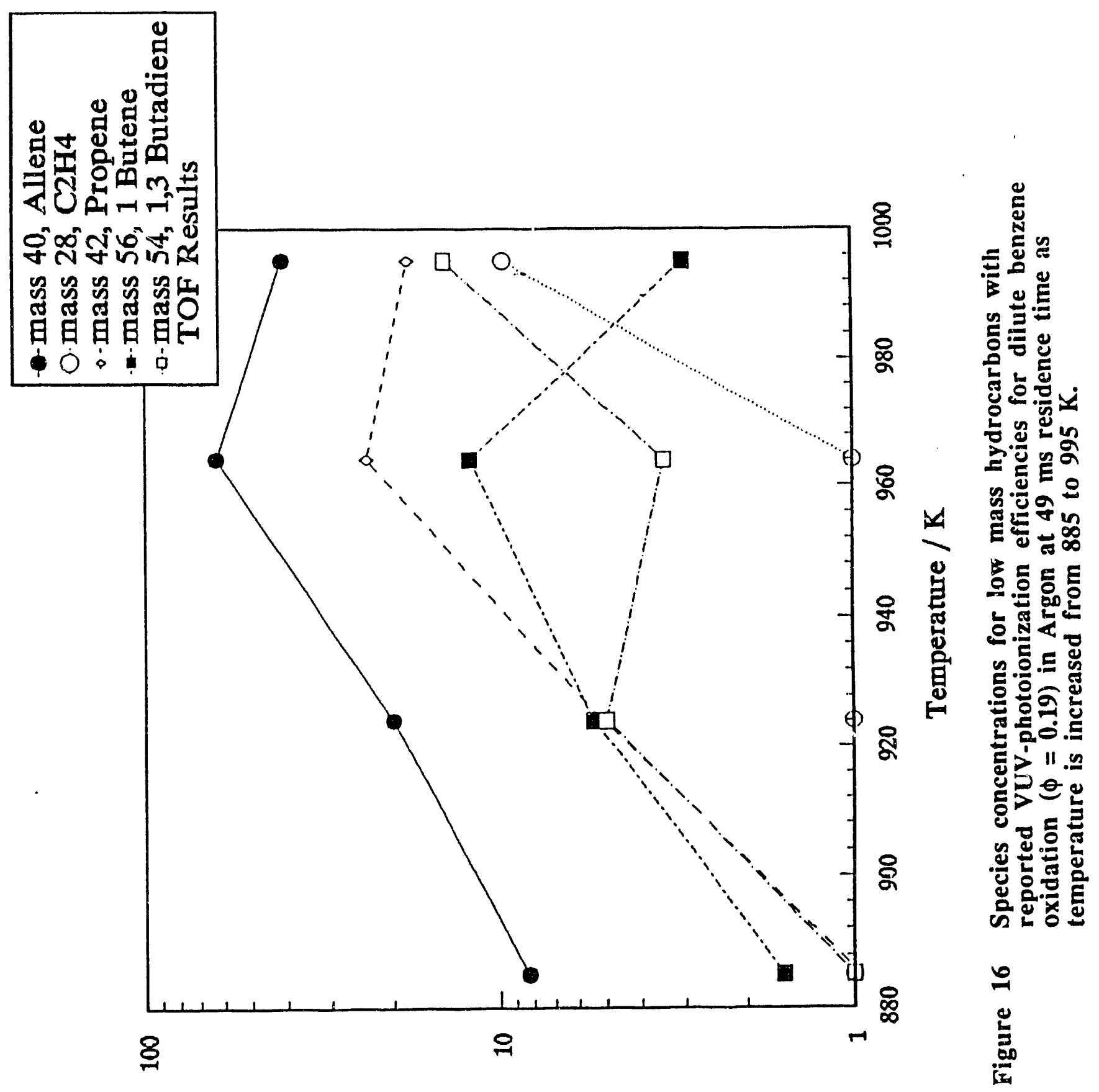

undd / uo!̣eมนวouos 


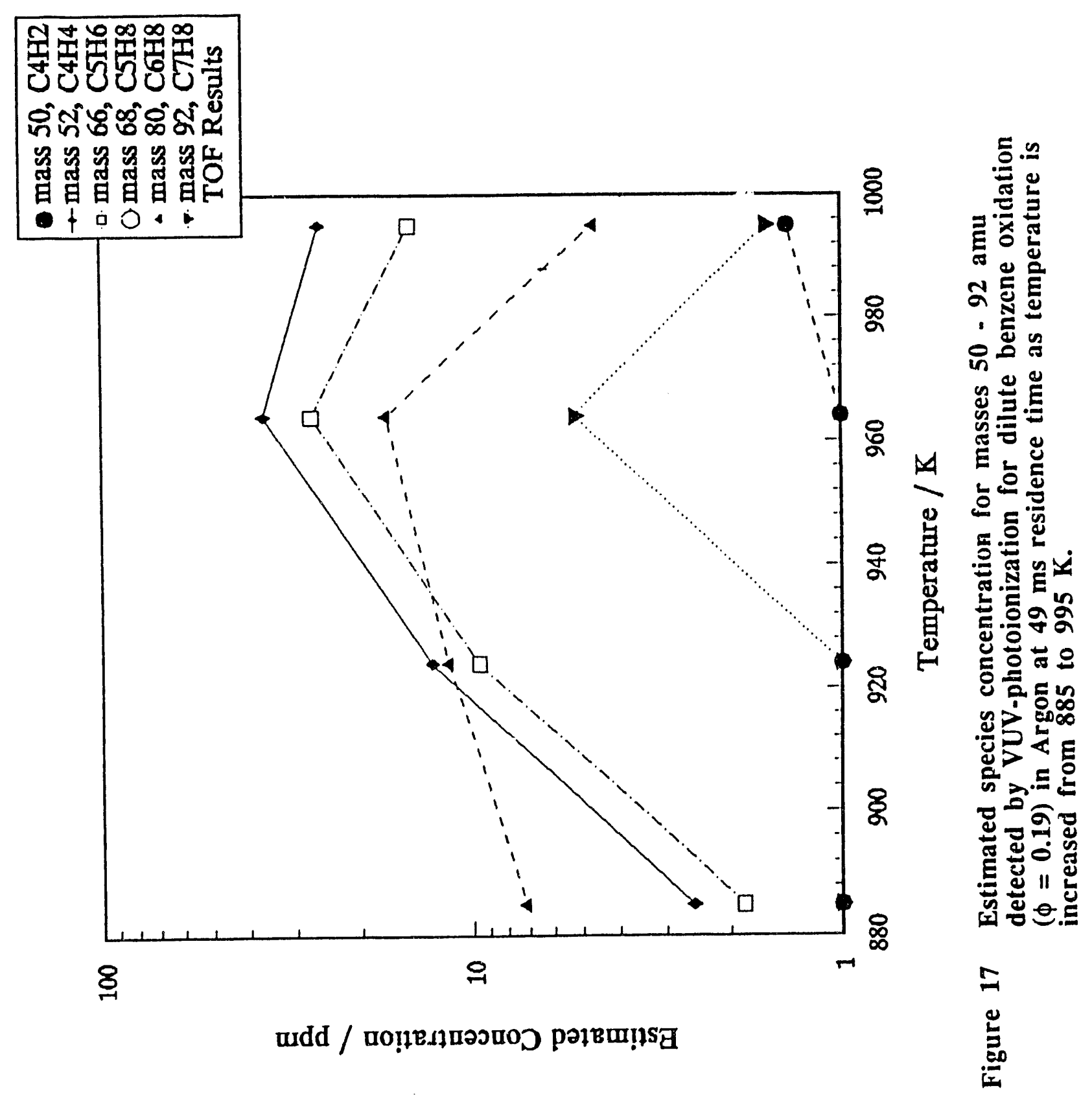




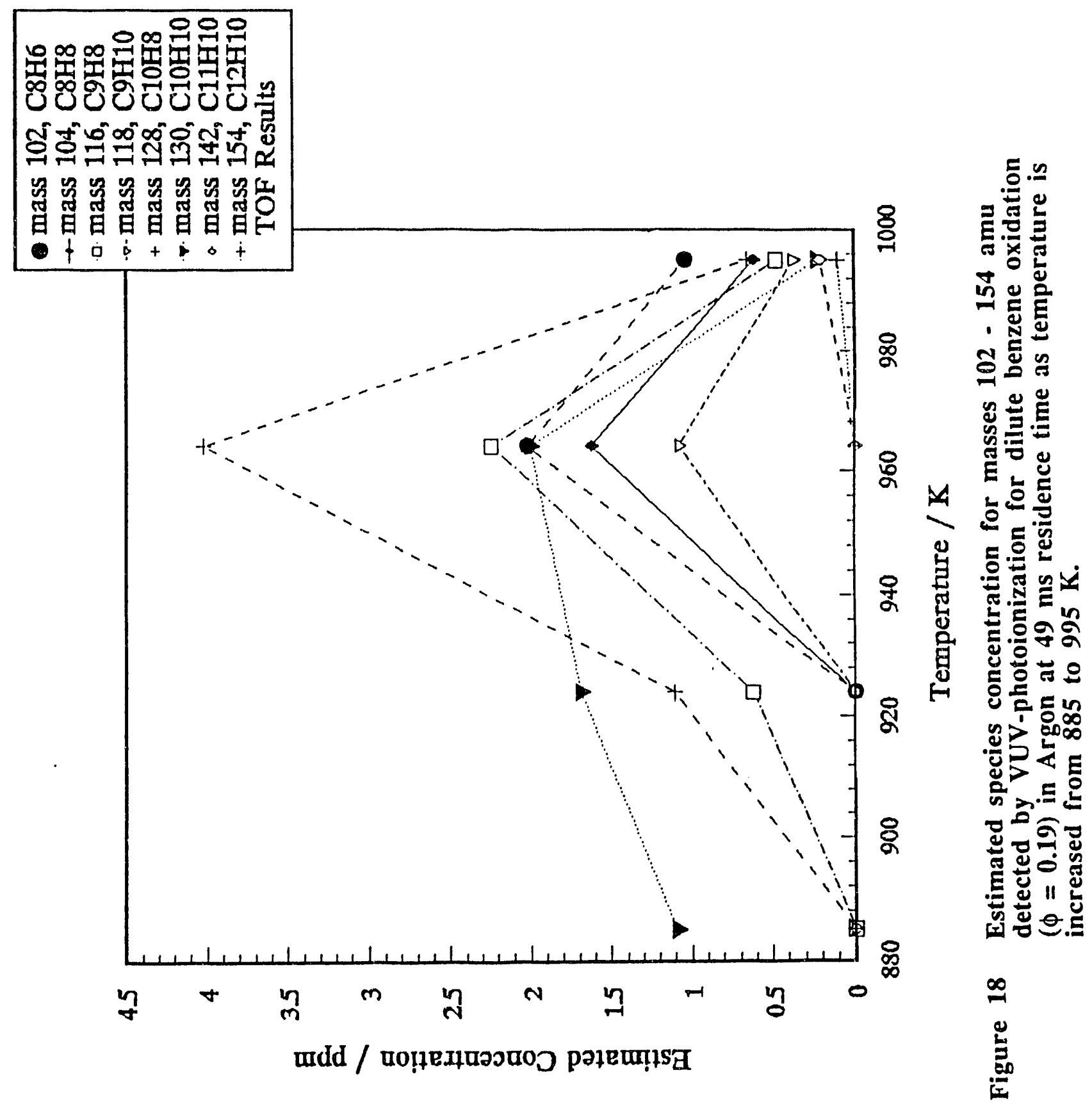




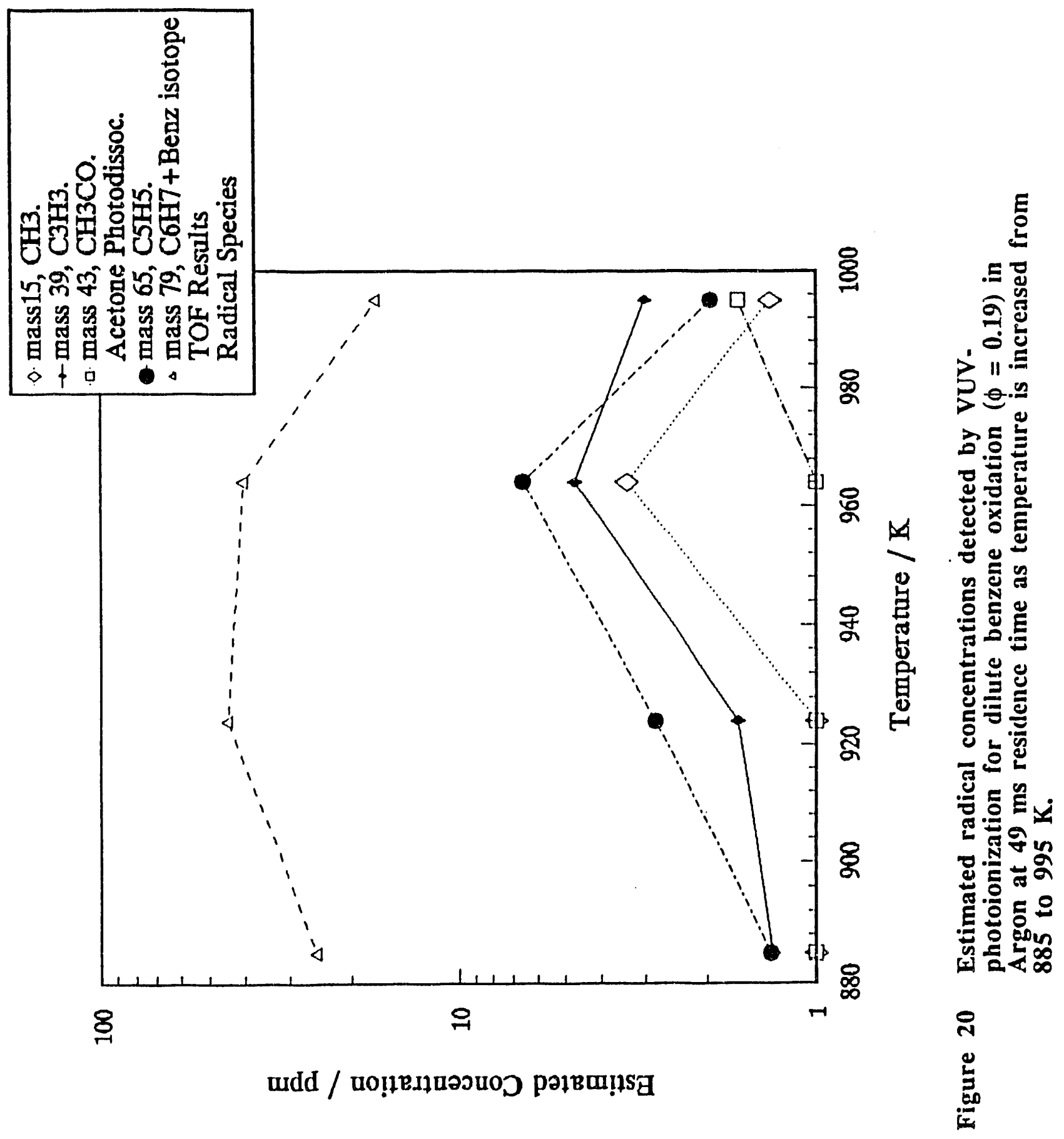




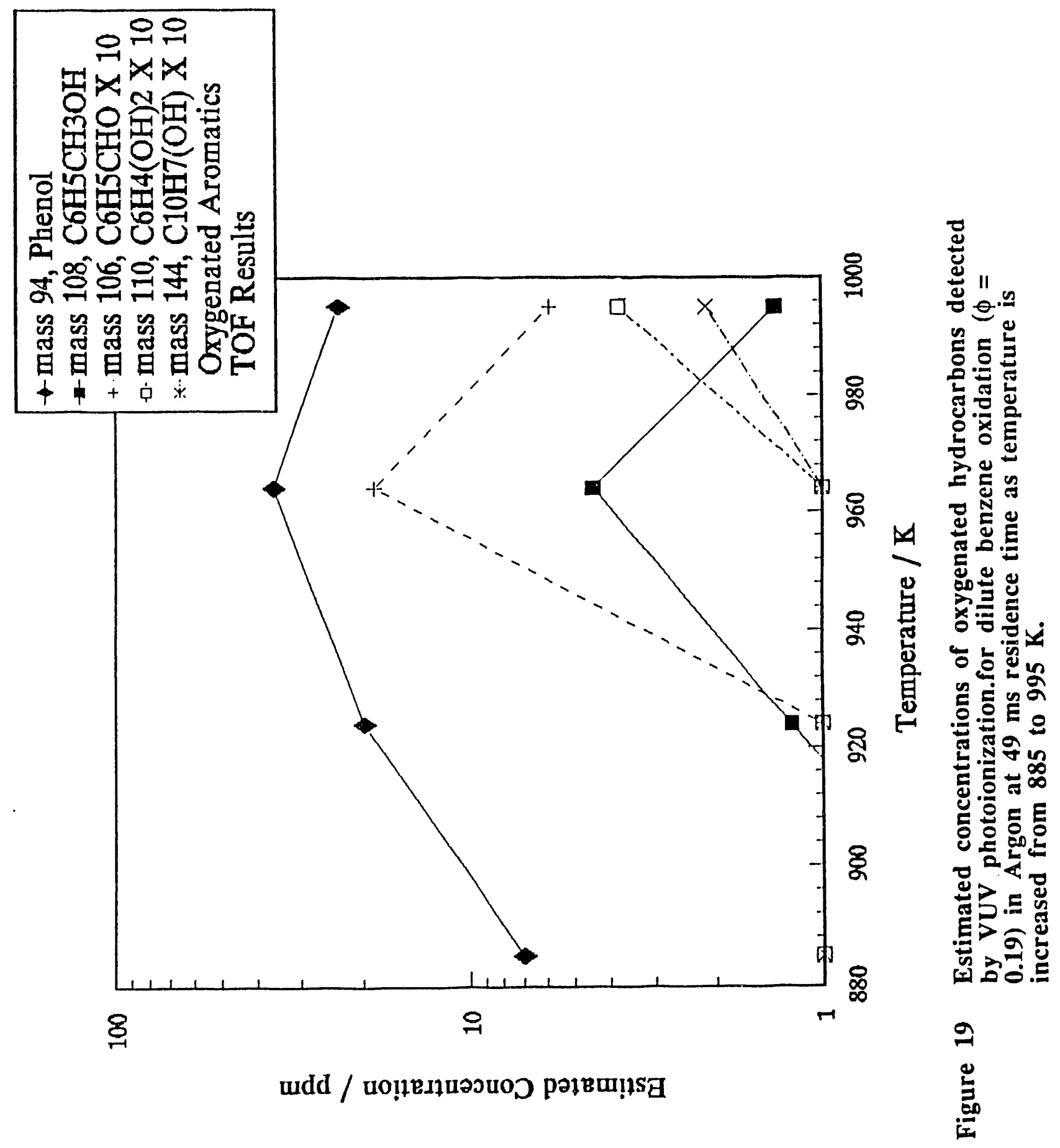




\subsection{REFERENCES}

Barnard, J.A. and Ibberson, V.J., Comb. and Flame, 9, 149 (1965) and references therein.

Bauer, S.H., 10th Symposium (International) on Combustion, The Combustion Institute, p. 511 (1965).

Benson, S.W., Shaw, R., J. Amer. Chem. Soc., 89 ,5351 (1967).

Blomquist, A.T., Verdol, J.A., J. Amer. Chem. Soc., 78109 (1956).

Boyle, J. PhD thesis, Studies of Pyrolysis and Oxidative Pyrolysis of Allene, Methyl Acetylene, Ethyl Acetylene and Butadiene using VUV Photoionization Mass spectrometry" Yale University (1991).

Boyle, J., Pfefferle, L.D., J. Phys. Chem., 94, 3336 (1990).

Boyle, J. and Pfefferle, L.D., Eastern States Combustion Institute Fall Meeting (1988).

Boyle, J., Pfefferle, L., LeBuo, J., Colson, S., in press Comb. Sci. Tech. (198')).

Boyle, J., Pfefferle, L.D., Gulcicek, E.E., and Colson, S.D., Rev. Sci. Instrurn., 62(2), 323 (1991).

Bruinsma, O.S.L., Moulijn, J.A., Fuel Processing Technology, 18, 213 (1988).

Calcote, C.. "The Role of Ions in Soot Formation", 2nd Workshop on Mechanisms and Models of Soot Formation, Heidelberg (1991).

Colson, S., Nuclear Instruments and Methods in Physics Research, B27, 130 (1987).

Colson, S., Frontiers of Laser Spectroscopy of Gases, p.309 - 324 (1988).

Colussi, A.J., Zabel, F., and Benson, S.W., Int. J. Chem. Kin. 9, 161 (1977).

D'Alessio, A., "Spectroscopic and chemical characterization of soot inception processes in premixed laminar flames at atmospheric pressure", 2nd Workshop on Mechanisms and Models of Soot Formation, Heidelberg (1991).

Eland, J. H. D., "Photoelectron Spectroscopy: An Introduction to Ultraviolet Photoelectron Spectroscopy in the Gas Phase", 2nd ed., Butterworths, London (1984).

Gajewski, J.J., Shih, C.N., J. Amer. Chem. Soc., 94, 1675 (1972).

Geno, P.W., MacFarlane, R.D., Int. J. Mass Spectrom. Ion Processes, 92, 195 (1989).

Glassman, I., "Combustion", Academic Press, Inc., Orlando, Florida, 1987

Glassman, I., 22nd Symposium (International) on Combustion, The Combustion Institute, p. 295 (1988).

Hidaka, Y., Namamura, A., Miyauchi, A., Shiraishi, T., Kawano, H., Int. H. Chem. Kinet., 21, 643 (1989).

Homann, K.H., Wagner, H. Gg., $11^{\text {th }}$ Symposium (International) on Combustion, The Combustion Institute, p. 371 (1967). 
Kern, R.D., Wu, C.H., J. Phys. Chem., 91, 6291 (1987).

Kiefer, J.H., Mudipalli, P., Sidhu, S.S., Kern, R.D., Xie, K., Chen, H., Twenty-Fourth Combustion Symposium, Colloquium on Reaction Kinetics (1991).

Kimura, K., Katsumata, S., Achiba Y., "Handbook of HeI Photoelectron Spectra of Fundamental Organic Molecules", Japan Scientific Societies Press, Tokyo, Halsted Press, N.Y., 1981.

Koizumi, H., J. Chem. Phys., 95 (8), 5846 (1991).

Koizumi, H., Yoshimi, T., Shinsaka, K., Ukai, M., Morita, M.,Hatano, Y., Yagashita, A., and Ito, J, J. Chem. Phys., 82, 4856 (1985), and other Koizumi and coworkers, e. g. Radiat. Phys. Chem., 43, 87 (1989), Radiat. Phys. Chem., 32, 111 (1988).

Lam, F.W., Howard, J.B., Longwell, J.P., 22 $2^{\text {nd }}$ Symposium (International) on Combustion, The Combustion Institute, p. 323 (1988.)

Longwell, J.P., $19^{\text {th }}$ Symposium (International) on Combustion, The Combustion Institute, p. $1339(1982)$.

McMillen, D.F., Trevor, P.L., Golden, D.M., J. Am. Chem. Soc., 102, 7400 (1980).

Meinert, R.N., Hurd, C.D., J. Amer. Chem. Soc., 52, 4540 (1930).

Person J. C., and Nicole P. P., J. Chem. Phys., 53, 1767 (1970),J. Chem. Phys., 49, 5421 (1968), J. Chem. Phys., 55, 3390 (1971).

Pfefferle, L.D., Boyle, J., Colson, S., Eastern States Combustion Meeting (1985);

Pfefferle, L.D., Boyle, J., Lobue, J., Colson, S., Combust. Sci. Tech., 70, 187 (1990).

Ritter, E., Bozzelli, J., THERM: Thermo Property Estimation for Radicals and Molecules, Dept. of Chemistry, New Jersey Institute of Technology.

Santoro, R., "Concentration and temperature effects on soot formation in diffusion flames", $2^{\text {nd }}$ Workshop on Mechanisms and Models of Soot Formation, Heidelberg (1991).

Sarofim, A., "Pathways to Soot Formation in Coal Combustion", 2nd Workshop on Mechanisms and Models of Soot Formation, Heidelberg (1991).

Stein, S.E., Walker, J.A., Suryan, M.M., Fahr, A., Twenty-Third Symposium on Combustion, The Combustion Institute, p. 85 (1989).

Stenhagen, E., Abrahamsson, S., and McLafferty, F.W., Eds. Atlas of Mass Spectral Data, Interscience, New York, I, 9 (1969).

Strauss C. E. and Funk, D. J., Optics Letters, 16(15), 1192 (1991).

Venkat, C., Brezinsky, K. and Glassman, I., 19th Symposium (International) on Combustion, The Combustion Institute, 1982, pp. 143-152.

Wagner, H.Gg., "Particulate Carbon Formation During Combustion", ed. D.C. Seigla and G.W. Smith, Plenum Press (1981). 
Wagner, H. Gg., Jander: "Pressure dependence of formation of soot and PAH in sooting flames", $2^{\text {nd }}$ Workshop on Mechanisms and Models of Soot Formation, Heidelberg (1991).

Weinstein, B., Fenselau, A.H., J. Chem. Soc., Section C, 368 (1967).

Westmoreland, P.R., Dean, A.M., Howard,J.B., Longwell, J.P., J. Phys. Chem., 93, 8171 (1989).

Westmoreland, P.R., Poster presentation, 23rd Symposium (Intl.) on Combustion (1990).

\subsection{HIGHLIGHT ACCOMPLISHMENTS}

Although many of the project highlights are described in detail above, several of the most important general accomplishments are noted below:

1. Demonstration of VUV-MS as a powerful technique for the analysis of stable and labile hydrocarbon species in a complex mixture.

2. Development of correlations for VUV photoionization yields based on PES data is continuing. This work will be summarized in the final report as a means for obtaining semi-quantitative detection of hydrocarbon radicals without direct calibration of each species.

3. Confirmation of benzene oxidation and pyrolysis kinetic mechanisms including semiquantitative measurement of cyclohexadienyl radical concentration.

4. Experimental observation of biaryl and other $\mathrm{PAH} / \mathrm{PAH}$ reaction pathways as important steps in the soot growth mechanism.

\subsection{ARTICLES AND PRESENTATIONS}

The PhD Thesis "The Detection of Soot Precursors from a Microjet Reactor Using Vacuum Ultraviolet Photoionization Mass Spectrometry" of the graduate student has been drafted and will be defended in $1 / 93$.

Pfefferle, L.D. "Higher Hydrocarbon Growth During Benzene Pyrolysis" abstract for 1992 Annual AIChE Meeting, Miami, Fl. 11/92.

Boyle, J., Pfefferle, L., Lobue, J., Colson, S., "Microreactor Coupled with Laser-Generated VUV Photoionization for Pyrolysis and Combustion Studies: Pyrolysis of Ethyl Acetylene,"Combust. Sci. and Tech.. Vol. 70, pp. 187-203.

Boyle, J., Pfefferle, L., Gulcicek, E. E., Colson, S., "Laser-driven electron ionization for a VUV photoionization time-of-flight mass spectrometer," Rev. Scl. Instrum.. Vol. 62(2), pp.323-333.

Pfefferle, L. D., "Higher Hydrocarbon Production During Allene Pyrolysis", Presentation at the ACS Annual Meeting, New York, NY, August, 1991.

Pfefferle, L. D., "Aromatic Ring Formation During Allene Pyrolysis", Presentation at the AIChE Annual Meeting, Los Angeles, CA, December, 1991.

Pfefferle, L. D., "VUV Ionization Mass Spectrometry as an Incinerator Diagnostic Tool", Presentation at the Second International Congress on Toxic Combustion By-Products: Formation and Control", Salt Lake City, UT, March, 1991. 


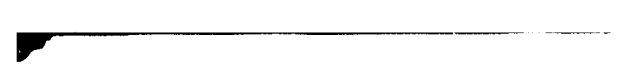

$\tau$
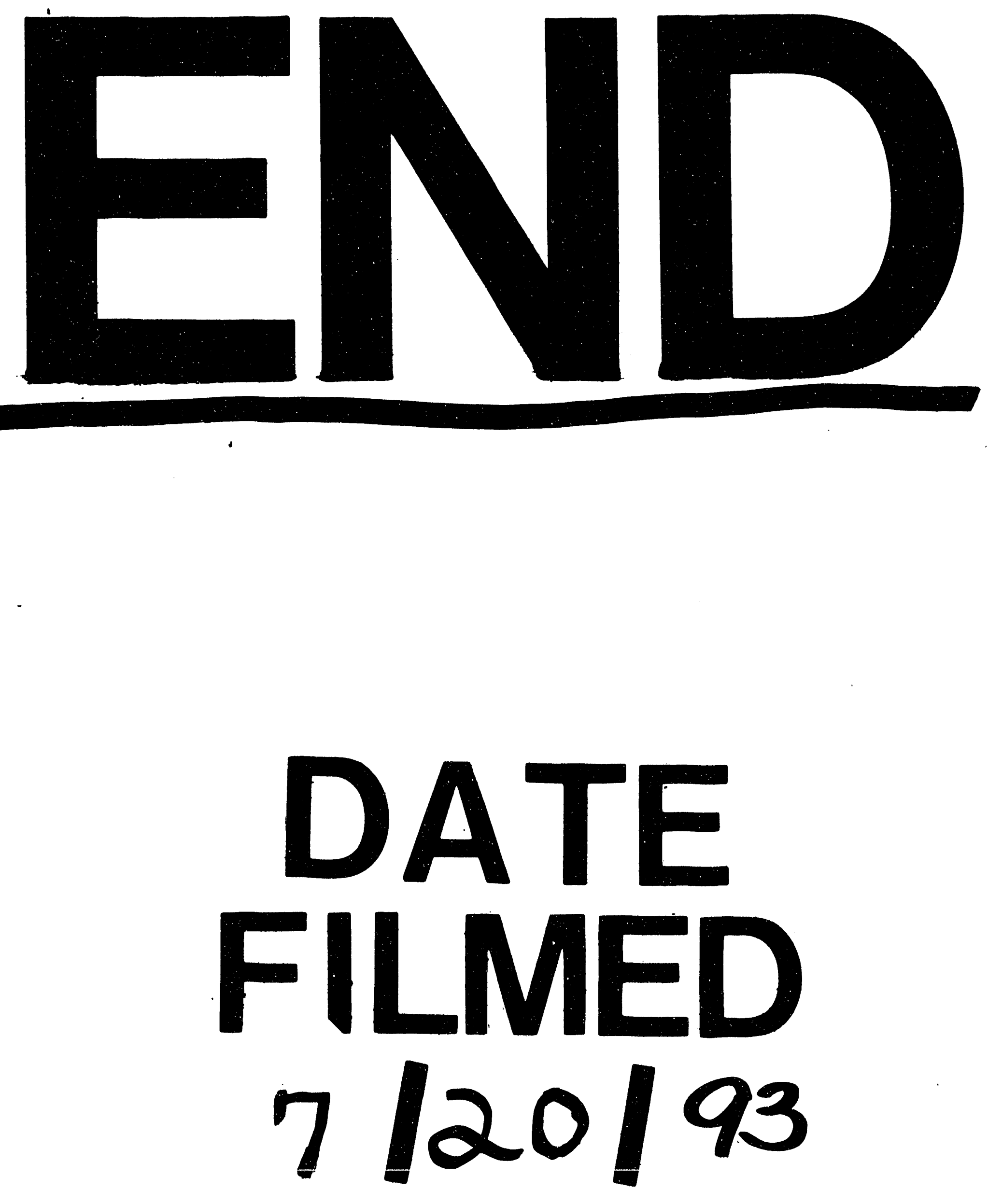
